\title{
Particle Morphology and Density Characterization by Combined Mobility and Aerodynamic Diameter Measurements. Part 1: Theory
}

\author{
Peter F. DeCarlo, ${ }^{1,2}$ Jay G. Slowik, ${ }^{4}$ Douglas R. Worsnop, ${ }^{5}$ Paul Davidovits, ${ }^{4}$ \\ and Jose L. Jimenez ${ }^{2,3}$ \\ ${ }^{1}$ Program in Atmospheric and Oceanic Sciences (PAOS), University of Colorado at Boulder, \\ Boulder, Colorado, USA \\ ${ }^{2}$ Cooperative Institute for Research in the Environmental Sciences (CIRES), University of Colorado \\ at Boulder, Boulder, Colorado, USA \\ ${ }^{3}$ Department of Chemistry and Biochemistry, University of Colorado at Boulder, Boulder, Colorado, USA \\ ${ }^{4}$ Boston College, Department of Chemistry, Chestnut Hill, Massachusetts, USA \\ ${ }^{5}$ Center for Aerosol and Cloud Chemistry, Aerodyne Research, Inc., Billerica, Massachusetts, USA
}

Different on-line submicron particle sizing techniques report different "equivalent diameters." For example, differential mobility analyzers (DMAs) report electrical mobility diameter $\left(d_{m}\right)$, while a number of recently developed instruments (such as the Aerodyne aerosol mass spectrometer, or AMS) measure vacuum aerodynamic diameter $\left(d_{v a}\right)$. Particle density and physical morphology (shape) have important effects on diameter measurements. Here a framework is presented for combining the information content of different equivalent diameter measurements into a single coherent mathematical description of the particles. We first present a review of the mathematical formulations used in the literature and their relationships. We then show that combining $d_{m}$ and $d_{v a}$ measurements for the same particle population allows the placing of constraints on particle density, dynamic shape factor $(\chi)$, and fraction of internal void space. The amount of information that can be deduced from the combination of $d_{m}$ and $d_{v a}$ measurements for various particle types is shown. With additional measurements and/or some assumptions, all relevant parameters can be determined. Specifically, particle mass can be determined from $d_{m}$ and $d_{v a}$ measurements if the particle density is known and an assumption about $\chi$ is made. Even if $\chi$ and density are not known, particle mass can be estimated within about a factor of 2 from $d_{m}$ and $d_{v a}$ measurements alone. The mass of a fractal particle can also be estimated under certain conditions. The meaning of various definitions

Received 2 July 2004; accepted 29 October 2004.

We thank Prof. Rick Flagan of Caltech for guiding our first steps in this area of research a few years ago. We are also grateful to Dan Imre and the AMS users' community for helpful discussions. This research was supported by NASA grant NNG04GA67G.

Address correspondence to Jose L. Jimenez, Department of Chemistry and CIRES, University of Colorado-Boulder, UCB 216 CIRES Bldg., Room 318, Boulder, CO 80309-0216, USA. E-mail: jose. jimenez@colorado.edu of "effective density" used in the literature is placed in the context of the theory. This theoretical framework is applied to measurements of fractal (soot-like) particles by using experimental results from the literature as additional constraints.

\section{INTRODUCTION}

Aerosol particles have important effects on human health, climate, regional visibility, and the deposition of acidic and toxic substances. Aerosols also have significant pharmaceutical and industrial applications. Because the properties of particles with respect to each of these issues are strongly affected by particle size, many instruments have been developed to measure the concentration of particles (e.g., number, mass, or chemical species concentration) as a function of particle size (Jayne et al. 2000; Baron et al. 2001a; Flagan 2001; Wexler and Johnston 2001).

Particles that deviate from the ideal characteristics of standard density $\left(1000 \mathrm{~kg} \mathrm{~m}^{-3}\right.$ or $\left.1.0 \mathrm{~g} \mathrm{~cm}^{-3}\right)$ and spherical shape have pronounced effects on particle sizing methods. Atmospheric aerosol particles are often nonspherical. For example, in the South Eastern Aerosol and Visibility Study, about 10\% of the particles in the 200-800 $\mathrm{nm}$ range were nonspherical (Dick et al. 1998). Soot aggregates are a type of non-spherical particles that is almost always found in the ambient aerosol (Katrinak et al. 1993). Soot or "black carbon" particles are aggregates of individual spherules produced by combustion, and are often termed fractal. Diesel engines in particular emit large amounts of soot particles. In addition to their importance in the atmosphere, the study of aggregate particles is of significant interest in the 
pharmaceutical industry as well as in the industrial production of nanoparticles. Aggregate particles have also been observed as compact shapes (Stober 1972). The physical and chemical characterization of nonspherical and fractal particles is an important area of current aerosol research (Friedlander and Pui 2004). This work focuses on the effect of particle shape and density on the methods of sizing aerosols through their effects on particle drag and inertia. A separate effect of particle shape (not discussed here) is due to lift forces on particle beams for irregular particles (Liu et al. 1995a; Jayne et al. 2000; Huffman et al. 2004). This article extends shape characterization of particles to the free molecular regime, and the companion article demonstrates the application of this framework to laboratoryproduced soot particles (Slowik et al. 2004). In the next section we review the definitions of the various equivalent diameters and other parameters used to describe the particles and explore their relationships. The following section presents a brief overview of the literature on particle shape and density estimation. The next section explores the information obtainable from combined mobility and aerodynamic diameter measurements. The last section focuses on the special case of aggregate particles. In this work we show that the combination of mobility and vacuum aerodynamic diameter measurements can provide constraints on the density, dynamic shape factor, and fraction of void spaces of the particles. We illustrate the theory by discussing a series of particle types. Two additional independent measurements (one of which could be particle mass obtained with an aerosol particle mass analyzer (APM) or the Aerodyne aerosol mass spectrometer (AMS)) allow the solution of the system and the determination of all the unknown parameters in near real-time. These parameters are particle mass, volume, dynamic shape factor, and density. In the absence of additional measurements, particle mass can still be estimated within about a factor of two for irregular particles from $d_{m}$ and $d_{v a}$ measurements alone.

\section{EQUIVALENT DIAMETERS AND RELATED CONCEPTS}

In this section we define the terms and notations that will be used throughout this article. Given the numerous definitions of particle diameters and different notations used in the literature, there is a need to make the definitions unambiguous for this work and to systematize the relationships between the different definitions. If particles are spherical and their material density is known, often these relations are trivial or simply a function of material density. When particles are nonspherical, contain void spaces, or when their material density is not known, the relationships between the different diameters become more complex and often underdetermined. This section introduces a framework that can be used for combining the information content of different diameter measurements into a single coherent mathematical description of the particles.

We begin with a list of notation followed by definitions of the various diameters used in characterizing aerosols.

\author{
Notation \\ $\lambda$ \\ $\mathrm{Kn}$ \\ $d_{p}$ \\ $d_{v e}$ \\ $d_{e}$ \\ $d_{m e}$ \\ $d_{m}$ \\ $d_{a}$ \\ $d_{c a}$ \\ $d_{t a}(\mathrm{Kn})$ or $d_{t a}$ \\ $d_{v a}$ \\ $d_{a d j}$ \\ $d_{A}$ \\ $d_{p p}$ \\ $C_{c}$ \\ $\chi$ \\ $\chi_{c}$ \\ $\chi_{t}(\mathrm{Kn})$ or $\chi_{t}$ \\ $\chi_{v}$ \\ S \\ $m_{p}$ \\ $\rho_{0}$ \\ $\rho_{m}$ \\ $\rho_{p}$ \\ $\rho_{\text {eff }}$ \\ $D_{f}$ \\ $V_{p}$ \\ $V_{m}$ \\ $V_{\text {void }}$ \\ $V_{a}$ \\ $\omega$ \\ $\delta$ \\ $\omega^{\prime}$ \\ mean free path of gas molecules \\ Knudsen number \\ physical or geometric diameter \\ volume equivalent diameter \\ envelope equivalent diameter (same as $d_{v e}$ ) \\ mass equivalent diameter \\ electrical mobility diameter \\ aerodynamic diameter (in any flow regime) \\ continuum regime aerodynamic diameter \\ transition regime aerodynamic diameter \\ vacuum aerodynamic diameter (also known as \\ free-molecular regime aerodynamic diameter) \\ adjusted sphere diameter (see section "Relation- \\ ship of $\chi$ to Flow Regime" below) \\ projected area diameter \\ physical diameter of a primary particle of an \\ aggregate \\ Cunningham slip correction factor \\ dynamic shape factor (in any flow regime) \\ dynamic shape factor (continuum regime limit) \\ dynamic shape factor (transition regime) \\ dynamic shape factor (vacuum or free molecular \\ regime limit) \\ Jayne shape factor \\ particle mass \\ standard density $\left(1 \mathrm{~g} \mathrm{~cm}^{-3}\right)$ \\ density of the material in the particle \\ particle density (see specific definition in Equa- \\ tion (5)) \\ effective or "apparent" density (see various def- \\ initions in section "Effective Density" below) \\ fractal dimension \\ particle volume (see specific definition in Equa- \\ tion (2)) \\ material volume (see specific definition in \\ Equation (1)) \\ void space volume \\ apparent volume \\ volume fraction of internal void spaces \\ parameter related to the fraction of internal void \\ spaces (see Equation (6)) \\ volume fraction of external pseudovoid spaces
}

\section{Equivalent Diameters}

Physical Diameter $\left(d_{p}\right)$. At the simplest level a particle can be characterized by its geometric or physical diameter. If the particle is spherical the meaning of this parameter is obvious, otherwise it does not have a precise meaning. Nonspherical (and sometimes nonstandard density) particles are generally characterized by equivalent diameters, defined as the diameter of a sphere, which with a given instrument would yield the same size measurement as the particle under consideration. 
Volume Equivalent Diameter $\left(d_{v e}\right)$. The volume equivalent diameter, also known as envelope equivalent diameter $\left(d_{e}\right)$, is defined as the diameter of a spherical particle of the same volume as the particle under consideration (Baron and Willeke 2001). For an irregular particle $d_{v e}$ is the diameter that the particle would have if it were melted to form a droplet while preserving any internal void spaces. Internal void spaces are empty regions of the particle that are isolated from the surrounding gas. Particles with internal voids can be encountered in some systems. For example, some aggregates from combustion sources can be relatively compact particles that have internal void spaces which are effectively isolated from the surrounding gas (Stober 1972; Kasper 1982a). Also, $d_{v e}$ has the advantage of being equal to $d_{p}$ for spherical particles (Hinds 1999). The volume equivalent diameter is the diameter to which we will reference all other equivalent diameters discussed in this article.

Mass Equivalent Diameter $\left(d_{m e}\right)$. The mass equivalent diameter is similar in concept to $d_{v e}$ but with the difference that $d_{m e}$ does not include internal voids. Therefore, for a particle with no internal voids $d_{m e}=d_{v e}$. If the particle contains internal voids, $d_{v e}>d_{m e}$ (Baron and Willeke 2001). This distinction is important because it leads to two parallel definitions of volume, density, and dynamic shape factor.

\section{Volume and Density}

Material Volume $\left(V_{m}\right)$. The material volume of a particle is the volume taken up by all of the solid and liquid material in the particle. In terms of $d_{m e}$ this volume is:

$$
V_{m}=\frac{\pi}{6} d_{m e}^{3} .
$$

Particle Volume $\left(V_{p}\right)$. The volume of the particle is determined by all material and void space enclosed within the particle envelope. In terms of $d_{v e}$ and the total volume of internal void spaces $\left(V_{\text {void }}\right), V_{p}$ is

$$
V_{p}=\frac{\pi}{6} d_{v e}^{3}=V_{m}+V_{v o i d}
$$

Material Density $\left(\rho_{m}\right)$ Material density is the average density of the solid and liquid material in the particle, and is expressed in terms of the particle mass $\left(m_{p}\right)$, material volume, and mass equivalent diameter as

$$
\rho_{m}=\frac{m_{p}}{V_{m}}=\frac{m_{p}}{\frac{\pi}{6} d_{m e}^{3}} .
$$

If several solid or liquid phases ( $a, b$, etc.) coexist in an individual particle, the material density is related to the density of the individual phases by

$$
\rho_{m}=\frac{m_{p}}{V_{m}}=\frac{\rho_{a} V_{a}+\rho_{b} V_{b}+\cdots}{V_{a}+V_{b}+\cdots}=\rho_{a} V F_{a}+\rho_{b} V F_{b}+\cdots .
$$

Here $\rho_{i}$ is the material density of phase $i, V_{i}$ is its volume, and $V F_{i}$ is its volume fraction in the particle.
Particle Density $\left(\rho_{p}\right)$. Particle density is referenced to the particle volume $\left(V_{p}\right)$ and volume equivalent diameter. Particle density is obtained when internal voids are included in Equation (4) as a phase with zero density:

$$
\rho_{p}=\frac{m_{p}}{V_{p}}=\frac{m_{p}}{\frac{\pi}{6} d_{v e}^{3}}=\frac{\rho_{a} V_{a}+\rho_{b} V_{b}+\cdots}{V_{\text {void }}+V_{a}+V_{b}+\cdots} .
$$

Note that $\rho_{p} \leq \rho_{m}$, with the equality being valid in the absence of internal voids.

Internal Void Fraction of a Particle. The fraction of internal voids in a particle is generally characterized by use of the parameter $\delta$, defined as (Baron et al. 2001b)

$$
\delta=\left(\frac{\rho_{m}}{\rho_{p}}\right)^{1 / 3} .
$$

By definition $\delta \geq 1$. The volume equivalent diameter, $d_{v e}$, can be related to $d_{m e}$ via Equation (6), yielding:

$$
d_{v e}=\delta \cdot d_{m e}
$$

We define the parameter $\omega$ as the volume fraction of internal void spaces

$$
\omega=\frac{V_{p}-V_{m}}{V_{p}}=1-\frac{1}{\delta^{3}} .
$$

The fraction of material volume of the particle is then

$$
\frac{V_{m}}{V_{p}}=\frac{1}{\delta^{3}}=1-\omega .
$$

\section{Flow Regimes}

Often diameters reported by different instruments can be related to the above equivalent diameters as functions of density, shape, and void fraction. However, in many instruments the measurements may also depend on the flow regime of the gas around the particle. This occurs because particles are often sized utilizing the ratio of drag force (exerted on the particle by the gas molecules) to some other force exerted on the particle, and the drag force can have different dependences on particle size and shape in the different flow regimes.

Knudsen Number (Kn). The flow regime of the gas around a particle is determined by the Knudsen number. Kn is defined as the ratio the mean free path of the gas molecules to the particle radius (Baron and Willeke 2001):

$$
\mathrm{Kn}=\frac{\lambda}{r}=\frac{2 \lambda}{d} .
$$

The limit of $\mathrm{Kn} \ll 1$ is referred to as the continuum regime flow where the gas can be thought of as a continuous fluid in its flow around the particle. The limit of Kn $\gg 1$ is called the free-molecular regime, where flow is described as a series of 
discrete "ballistic" collisions of the gas molecules with the particle. For intermediate values of $\mathrm{Kn}(0.1<\mathrm{Kn}<10)$, particles are said to be in the transition regime between continuum and free-molecular flow.

Drag in the Continuum Regime. In the simplest case, Stokes law governs the drag in the continuum regime, that is,

$$
F_{\text {drag }}=3 \pi \eta v d_{p}
$$

Here $\eta$ is the gas dynamic viscosity and $v$ is the velocity of the particle relative to the gas. Nonspherical particles experience more drag than their volume or mass equivalent spheres because they present a larger surface for interaction with the gas molecules. If the drag force is expressed as a function of the volume equivalent diameter, a correction factor must be used to account for the increased drag due to nonspherical shape (see section "Dynamic Shape Factor" below). If the particle Reynolds Number, $\operatorname{Re}_{p}>0.1$ the drag force must be corrected for nonStokesian effects.

Drag in the Transition and Free-Molecular Regimes. Acorrection to the drag equation must be introduced to account for the reduction in drag that occurs when the relative velocity of the gas at the particle surface is nonzero (Hinds 1999). The reduced drag is significant when the flow around the particle is outside the continuum regime $(\mathrm{Kn}>0.1)$. This correction is implemented via the Cunningham Slip Correction Factor, $C_{c}(\mathrm{Kn})$, which was parameterized by Allen and Raabe $(1982,1985)$ as:

$$
\begin{aligned}
C_{c}(\mathrm{Kn}) & =1+\mathrm{Kn}\left[\alpha+\beta \exp \left(-\frac{\gamma}{\mathrm{Kn}}\right)\right] \\
C_{c}(d) & =1+\frac{2 \lambda}{d}\left[\alpha+\beta \exp \left(-\frac{\gamma}{2 \lambda / d}\right)\right] .
\end{aligned}
$$

Here $\alpha, \beta$, and $\gamma$ are empirically determined constants specific to the system under analysis. For example, if the suspending gas is different than air at Normal Temperature and Pressure (NTP, $298 \mathrm{~K}$ and $1 \mathrm{Atm}$ ), then the parameter values vary accordingly (Rader 1990). Kn is defined in Equation (10), and $d$ is the particle diameter according to one of the definitions presented above. In general, the value of $C_{c}$ will be different for the different equivalent diameters of the same particle. Values of $\alpha, \beta$, and $\gamma$ have been determined for solid particles and oil droplets under normal atmospheric conditions. For solid particles $\alpha$ is $1.142, \beta$ is 0.558 , and $\gamma$ is 0.999 (Allen and Raabe 1985). For oil droplets $\alpha$ is $1.207, \beta$ is 0.440 , and $\gamma$ is 0.596 (Rader 1990). The asymptotic limits for the slip correction allow for smooth transition of the drag force between flow regimes. $C_{c}$ asymptotically approaches 1 in the continuum regime limit (ignoring the Cunningham correction for $\mathrm{Kn}=0.1$ results in an error of approximately $10 \%$ ) and in the free-molecular regime Equation (12) becomes

$$
C_{c}(d) \approx \frac{2 \lambda}{d}(\alpha+\beta)
$$

Applying Equation (13) to a particle $1 \mu \mathrm{m}$ in diameter at 1.5 Torr (typical AMS conditions as described below) results in a maximum error of $0.6 \%$ when compared to the value calculated by Equation (12). The error decreases as the diameter decreases or $\lambda$ increases.

Adding the slip correction to Equation (11), the equation for drag on a sphere in any flow regime (Baron and Willeke 2001) is

$$
F_{\text {drag }}=\frac{3 \pi \eta v d_{p}}{C_{c}\left(d_{p}\right)} .
$$

Thus the drag force in the transition and free-molecular regimes is smaller than the drag calculated in the continuum regime (Equation (11)).

\section{Dynamic Shape Factor $(\chi)$}

Definition of the Dynamic Shape Factor. A second correction must be introduced into Equation (11) to account for the increased drag on a particle due to nonspherical shape. This correction is called the dynamic shape factor $(\chi)$ and was first introduced by Fuchs (1964). It is defined as the ratio of the resistance force (typically the drag force) on the nonspherical particle to the resistance force on its volume equivalent sphere, when both move at the same relative velocity with respect to the gas (Hinds 1999):

$$
\chi=\frac{F_{D}^{p}}{F_{D}^{v e}} .
$$

The dynamic shape factor is almost always greater than one for irregular particles and equal to one for spheres. The dynamic shape factor is used with the slip correction factor as an additional correction to Equation (11). The general equation for drag in any flow regime is (Baron and Willeke 2001)

$$
F_{d r a g}=\frac{3 \pi \eta v \chi d_{v e}}{C_{c}\left(d_{v e}\right)} .
$$

Effect of Particle Orientation on $\chi$. In some cases particles with streamlined or nonsymmetrical shapes may adopt a preferred orientation in the flow (Dahneke 1973a; Hinds 1999; Baron et al. 2001b). In the case of streamlined particles it is possible that the dynamic shape factor attains values less than 1 (Hinds 1999). Orientation effects likely do not play a role in AMS sizing, where the particle is imparted a size-dependent velocity when the particle Reynolds number is $\sim 0.03$ or smaller (Zhang et al. 2002), and as stated in Hinds (1999) no alignment is expected for particle Reynolds numbers less than 0.1. Alignment of particles in a differential mobility analyzer (DMA) or scanning mobility particle sizer (SMPS) system can be a function of the charge location on the particle and strength of the electric field (Kousaka et al. 1996) and could play a role in measurements of some types of irregular particles (Baron et al. 2001b). Further research is needed to quantify the effects of orientation in these sizing techniques. 
Relationship of $\chi$ to Flow Regime. The value of $\chi$ can depend on the flow regime due to differences in the effect of the nonsphericity on drag in the different flow regimes and the use of $d_{v e}$ in $C_{c}$ on Equation (16) (Dahneke 1973a; Cheng 1991). Here we use the symbol $\chi$ (as a short for $\chi(\mathrm{Kn})$ ) for the general dynamic shape factor of a particle that accounts for the different flow regimes. In the limits of the continuum regime and freemolecular regime, $\chi$ asymptotically approaches unique values, termed $\chi_{c}$ and $\chi_{v}$, respectively. In the transition regime, the shape factor will be denoted as $\chi_{t}$ or $\chi_{t}(\mathrm{Kn})$. Note that $\chi_{t}$ will change with gas pressure for a given particle due to this dependence on $\mathrm{Kn}$.

Dahneke (1973a, b) numerically calculated drag on regularly shaped particles in the continuum and free-molecular regimes. Translating his calculated drag results for bodies of revolution and cubes into dynamic shape factors (see method in Appendix A), we can shed some light on the relationship between $\chi_{c}$ and $\chi_{v}$. Dynamic shape factors for doublets and triplets of spheres have also been reported in both the continuum and freemolecular regimes (Kousaka et al. 1996; Baron et al. 2001b). Figure 1 displays these results for a comparison between $\chi_{c}$ and $\chi$. It is expected that values of $\chi_{t}(\mathrm{Kn})$ in the transition regime change smoothly between these two extremes (Dahneke 1973c). A special case for aggregate particles is discussed later. Figure 1 neglects orientation effects, and $\chi_{c}$ and $\chi_{v}$ are calculated using values averaged over all orientations. Consequently values of $\chi$

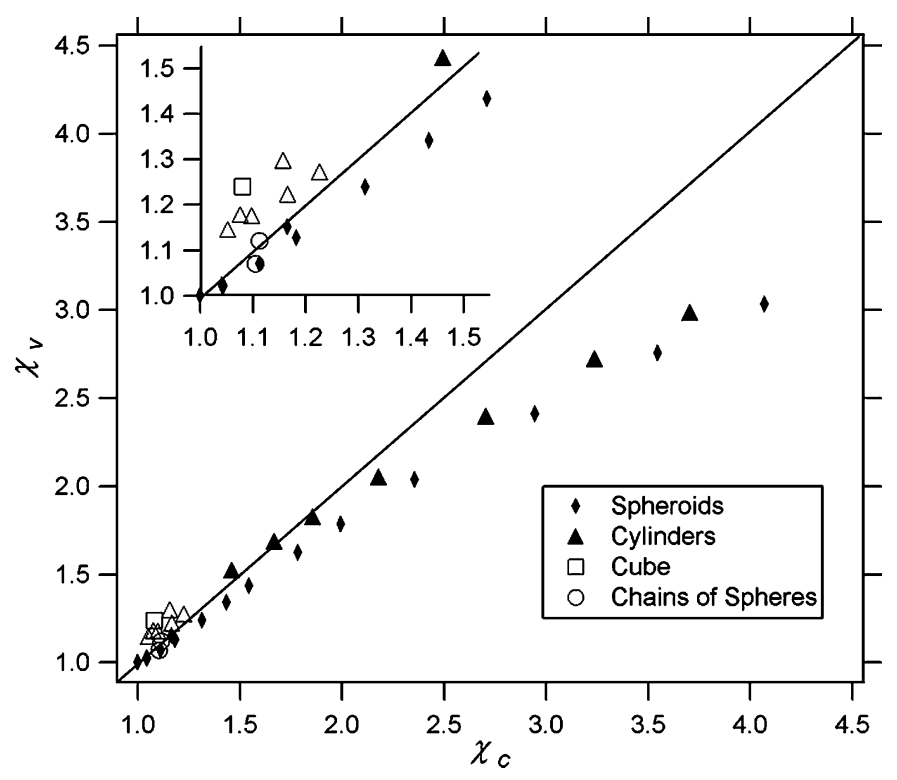

Figure 1. Comparison of $\chi_{c}$ and $\chi_{v}$ values for several simple particle shapes calculated from the results of Dahneke (1973a, b, c) by the method shown in Appendix A. Values for chains of spheres were taken from Chan and Dahneke (1981) and Kousaka et al. (1996). Open symbols indicate when experimental results for continuum flow drag were used, with all free-molecular flow values numerically calculated. Filled symbols identify points in which both $\chi_{c}$ and $\chi_{v}$ were calculated numerically. could be different in cases when particles can have a preferred orientation in the flow (Dahneke 1973a). Orientation effects are not explicitly treated in this article, but the mathematical formulation is still valid if orientation-specific shape factors such as those given by Dahneke (1973a, b) are used.

Figure 1 suggests that for small values of $\chi(<2), \chi_{c} \approx \chi_{v}$ is a fair approximation. Note that this comparison is based on limited experimental and numerical results, and more research into this subject for additional particle types is necessary to verify or discount this relationship. Figure 1 is meant to present the range of $\chi_{c}$ and $\chi_{v}$ values for the same particles based on current knowledge.

Estimation of $\chi_{t}(\mathrm{Kn})$ in the Transition Regime. Dahneke introduced the adjusted sphere formulation to allow the estimation of drag forces on a particle across flow regimes (Dahneke 1973a). In this formulation the adjusted sphere diameter $\left(d_{a d j}\right)$ replaces $d_{v e}$ in the slip correction factor in the calculation of drag (Equation (16)):

$$
F_{d r a g}=\frac{3 \pi \eta v \chi_{c} d_{v e}}{C_{c}\left(d_{a d j}\right)}
$$

$d_{a d j}$ is a calculated diameter that allows a smooth transition between the drag forces for the continuum regime to the freemolecular regime based on an asymptotic fitting of the drag forces in each extreme. In this formulation the dynamic shape factor used in Equation (17) does not change with flow regime; instead, it is held constant at $\chi_{c}$ and the adjusted sphere diameter compensates for the change to the shape factor. This formulation has been shown to be in good agreement with experimental data over the transition regime for limited particle types (Dahneke 1973c; Cheng 1991; Chen et al. 1993). At the asymptotic limit of the free molecular regime, Equations (13), (16), and (17) can be used to relate $d_{a d j}$ to our formulation as

$$
d_{a d j}=\frac{\chi_{v}}{\chi_{c}} d_{v e}
$$

Using Equations (16-18) we can estimate the dynamic shape factor in the transition regime as

$$
\chi_{t}(\mathrm{Kn})=\chi_{c} \frac{C_{c}\left(d_{v e}\right)}{C_{c}\left(\frac{\chi_{v}}{\chi_{c}} d_{v e}\right)} .
$$

Alternate Definition of the Dynamic Shape Factor Based on $d_{m e}$. Equation (15) is the most commonly used definition of the dynamic shape factor, i.e., the ratio of the drag forces for the actual particle and its volume-equivalent sphere moving at the same relative velocity with respect to the gas. It is also possible to define a dynamic shape factor based on the mass-equivalent diameter $\left(d_{m e}\right)$ rather than on $d_{v e}$ as

$$
\chi^{\prime}=\frac{F_{D}^{p}}{F_{D}^{m e}} .
$$


For a particle with no internal voids this new definition equals the dynamic shape factor $(\chi)$ as defined by Equation (15). However, for a particle with internal voids this definition deviates from the standard definition due to the difference between $d_{v e}$ and $d_{m e}$. Although the $d_{m e}$-based shape factor is also generally denoted as $\chi$ in the literature, here we will use the symbol $\chi^{\prime}$ in order to make the distinction clear. The general expression for the drag force when $\chi^{\prime}$ and $d_{m e}$ are used is

$$
F_{\text {drag }}=\frac{3 \pi \eta v \chi^{\prime} d_{m e}}{C_{c}\left(d_{m e}\right)} .
$$

Equating the expressions for particle drag based on $d_{v e}$ (Equation (16)) and $d_{m e}$ (Equation (21)) allows the separation of $\chi^{\prime}$ into two parts (Baron and Willeke 2001), one due to the external shape of the particle and one due to the particle void spaces:

$$
\chi^{\prime}=\chi \frac{d_{v e}}{d_{m e}} \frac{C_{c}\left(d_{m e}\right)}{C_{c}\left(d_{v e}\right)}=\chi \delta \frac{C_{c}\left(d_{m e}\right)}{C_{c}\left(\delta d_{m e}\right)} .
$$

The external particle shape component of the dynamic shape factor is captured by $\chi$ (sometimes denoted as $\kappa$ in the literature (e.g., Allen et al. 1979; Kasper 1982a)). The distinction between $\chi$ and $\chi^{\prime}$ in Equation (22) can be understood in the context of a particle with internal void spaces. In this case $d_{m e}<d_{v e}$, which is equivalent to saying $\rho_{p}<\rho_{m}$. Therefore, always $\chi^{\prime} \geq \chi$, with the equality being valid for particles without internal voids. Deviations from a $\chi^{\prime}$ value of 1 are not necessarily indicative of a nonspherical particle and may in fact be due mostly to void spaces within a nearly spherical particle. Note that equations involving $d_{v e}, \rho_{p}$, and $\chi$ below could instead be written in an alternate form using $d_{m e}, \rho_{m}$, and $\chi^{\prime}$. Care should be taken not to mix parameters from both formulations into the equations below. For example, if $\rho_{m}$ is used and the particles are known to have internal voids, then the formulation with $d_{m e}$ and $\chi^{\prime}$ should be used, otherwise an error will be introduced.

\section{Electrical Mobility Diameter $\left(d_{m}\right)$}

The electrical mobility diameter is the diameter of a sphere with the same migration velocity in a constant electric field as the particle of interest (Flagan 2001). Instruments such as the DMA and the SMPS measure $d_{m}$. This measurement is obtained via a force balance between the electrical force of a constant electric field on the net charges on the particle and the drag force experienced by the particle. The electrical force on the particle is:

$$
F_{\text {elec }}=n e E \text {. }
$$

Here $n$ is the number of charges on the particle, $e$ is the elementary unit of charge, and $E$ is the strength of the electric field. Under typical DMA conditions a particle reaches a terminal migration velocity extremely quickly, at which point the electrical and drag forces are equal and opposite.

The relationship between the volume equivalent diameter $\left(d_{v e}\right)$ and $d_{m}$ is obtained from the electrical mobility, $Z_{p}$, de- fined as the steady-state migration velocity of a particle per unit electric field strength. Applying this definition with the drag force in Equation (16) and the electrical force in Equation (23), we obtain

$$
Z_{p}=\frac{n e C_{c}\left(d_{v e}\right)}{3 \pi \eta \chi_{t} d_{v e}}=\frac{n e C_{c}\left(d_{m}\right)}{3 \pi \eta d_{m}} .
$$

Note that particles in the DMA are generally in the transition regime, thus the dynamic shape factor is represented by $\chi_{t}$ (as a shorthand for $\chi_{t}(\mathrm{Kn})$ ). Note that the charging probability for an irregular particle is different than that for its volume equivalent sphere (Rogak et al. 1993). From Equation (24), and assuming the particle and its volume equivalent sphere have the same charge, we obtain the commonly used relationship between $d_{v e}$ and $d_{m}$ :

$$
\frac{d_{m}}{C_{c}\left(d_{m}\right)}=\frac{d_{v e} \cdot \chi_{t}}{C_{c}\left(d_{v e}\right)} .
$$

For spherical particles, $d_{m}$ equals $d_{p}$ and $d_{v e}$. For nonspherical particles, $d_{m}$ is always greater than $d_{v e}$ because $\chi_{t}(\mathrm{Kn})>1$, and $C_{c}$ is a monotonically decreasing function of $d$. Note that for nonspherical particles $d_{m}$ is not unique but depends on the pressure at which the measurement is performed, due to the dependence of $\chi_{t}$ and $C_{c}$ on $\mathrm{Kn}$. Strictly speaking, we can define $d_{c m}, d_{t m}(\mathrm{Kn})$, and $d_{v m}$ to denote the mobility diameters in the continuum, transition, and free-molecular regimes, respectively. However, most measurements of $d_{m}$ are performed under pressures near $1 \mathrm{~atm}$, so in this article we will only use the symbol $d_{m}$ for the mobility diameter. However, it is important to take into account the effect of $\mathrm{Kn}$ on $d_{m}$ when the pressure in the DMA changes in the course of the experiment, as when sampling from aircraft.

In summary, mobility diameters increase with increasing deviation from a sphere. Figure 2 a illustrates the trajectories of spherical particles of different sizes in the electric field of a DMA. It also shows an irregular particle with identical volume as the smaller sphere that follows the same trajectory as the larger sphere. Note that the diameter of the sphere following the same trajectory as the irregular particle is the mobility diameter of the irregular particle. An irregular particle experiences a larger drag force but the same electrical force compared to its volume equivalent sphere, so it is "sized" as a mobility-equivalent sphere that is larger than its volume-equivalent sphere, i.e., for irregular particles, $d_{m}>d_{v e}$.

\section{Aerodynamic Diameter}

Aerodynamic Diameter $\left(d_{a}\right)$. The aerodynamic diameter is defined as the diameter of a sphere with standard density that settles at the same terminal velocity as the particle of interest. As will be discussed, the aerodynamic diameter depends on the flow regime. We will begin our discussion of $d_{a}$ with a general exploration of the concept and then proceed to define specific instances of $d_{a}$ in the continuum and free-molecular regimes. 


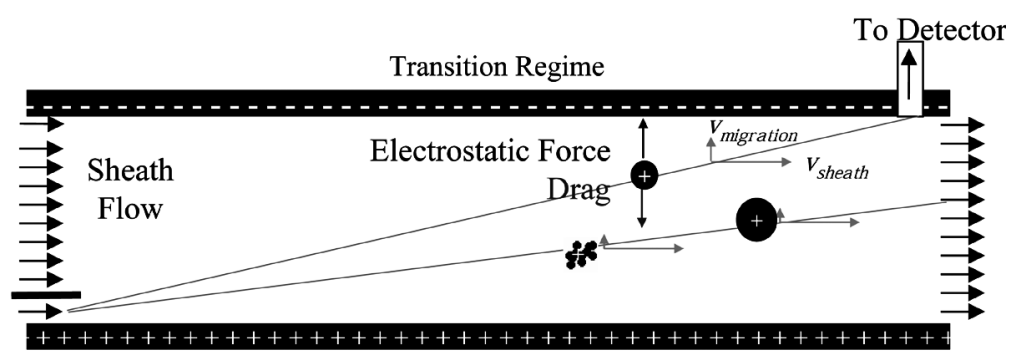

(a)

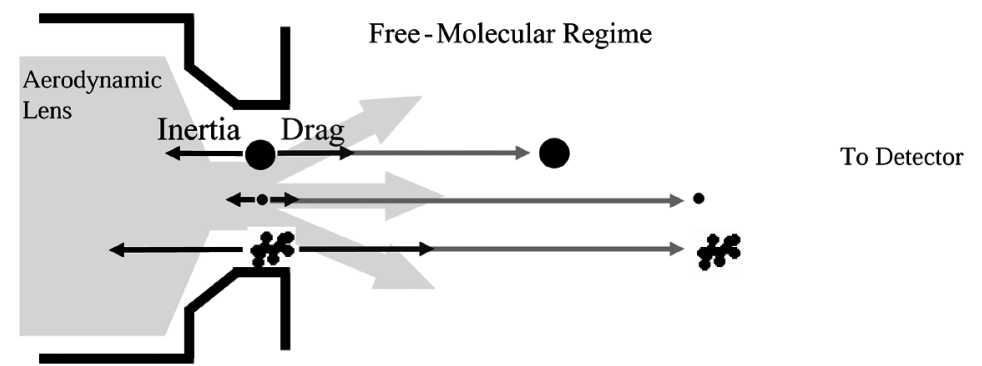

(b)

Figure 2. A schematic representation of the different diameter sizing measurements for (a) the DMA and (b) the AMS. In each case three particles are shown. In (a) the irregular particle has the same mass as the smaller sphere. Light gray arrows depict the velocity vectors for the horizontal (sheath flow) and the vertical (electrical migration). In (b) the irregular particle has the mass of the larger sphere. Gray arrows depict the velocity imparted to the particles at the nozzle expansion. All particles in the figure have standard density.

Terminal settling velocity $\left(v_{T S}\right)$ is a measure of the aerodynamic properties of the particle. Terminal velocity is obtained when the gravitational force $\left(F_{G}\right)$ is equal and opposite to the drag force from Equation (16):

$$
F_{G}=m_{p} g=\rho_{p} \frac{\pi}{6} d_{v e}^{3} g=\frac{3 \pi \eta v_{T S} d_{v e} \chi}{C_{c}\left(d_{v e}\right)}=F_{d r a g} .
$$

As stated above, the aerodynamic diameter is defined as the diameter of a standard density $\left(\rho_{o}\right)$ sphere with the same $v_{T S}$ as the particle. That is, in Equation (26), $\rho_{p}$ is standard density $\left(\rho_{0}\right), \chi=1$, and the diameter is $d_{a}$. The force balance in Equation (26) can then be expressed as

$$
F_{G}=m_{p} g=\rho_{0} \frac{\pi}{6} d_{a}^{3} \cdot g=\frac{3 \pi \eta v_{T S} d_{a}}{C_{c}\left(d_{a}\right)} .
$$

By definition, $v_{T S}$ is the same in Equations (26) and (27). Combining these two equations, we obtain (Hinds 1999)

$$
\begin{aligned}
d_{a} & =d_{v e} \sqrt{\frac{1}{\chi} \frac{\rho_{p}}{\rho_{0}} \frac{C_{c}\left(d_{v e}\right)}{C_{c}\left(d_{a}\right)},} \\
\rho_{0} d_{a}^{2} C_{c}\left(d_{a}\right) & =\frac{\rho_{p}}{\chi} d_{v e}^{2} C_{c}\left(d_{v e}\right) .
\end{aligned}
$$

The aerodynamic diameter increases with increasing particle density. Unlike for $d_{m}$ (e.g., when sizing with a SMPS system), particle density, and therefore particle composition, affects the sizing of the particles even if the physical morphology is the same. Spherical particles with $\rho_{p}>1.0 \mathrm{~g} \mathrm{~cm}^{-3}$ have a larger aerodynamic diameter than their geometric or physical diameter. The opposite is true for spheres with $\rho_{p}<1.0 \mathrm{~g} \mathrm{~cm}^{-3}$ (McMurry 2000). As with $d_{m}$, particle shape affects the relationship between $d_{a}$ and $d_{v e}$. Aerodynamic diameter decreases with increasing dynamic shape factor. For an irregular particle of unit (or lower) density, $d_{a}$ is always smaller than $d_{v e}$, while $d_{m}$ is larger than $d_{v e}$ (see section "Electrical Mobility Diameter" above). For particles of larger than standard density, $d_{a}$ may be smaller or larger than $d_{v e}$ depending on the relative values of $\rho_{p}$ and $\chi$.

Aerodynamic sizing is illustrated in Figure $2 \mathrm{~b}$ as it takes place in an Aerodyne AMS (Jayne et al. 2000; Jimenez et al. 2003c). Two spheres are shown, as is an irregularly shaped particle of standard density with the same volume as the larger sphere, and whose measured aerodynamic diameter would the same as that of the smaller sphere. In summary, for irregular particles of standard density, $d_{m}>d_{v e}>d_{a}$.

Highly irregular particle populations, such as diesel soot, will show significant differences in the size distributions measured simultaneously by mobility and aerodynamic techniques. These are not real discrepancies; instead, they merely capture the different dependence of both equivalent diameters on the fundamental particle properties, and can be used to yield information about the particle population with the methods presented below. This phenomenon has been observed in the measurement of ambient aerosol (Chakrabarti et al. 2004; Zhang et al. 2004a) 


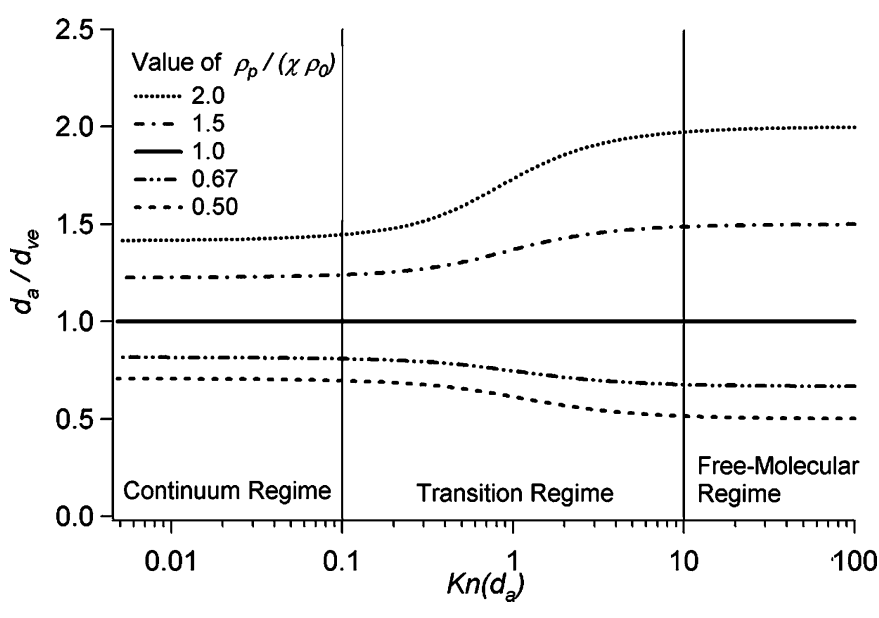

Figure 3. The ratio of $d_{a}$ to $d_{v e}$ as a function of $\mathrm{Kn}$ (with the assumption $\chi_{c} \approx \chi_{t} \approx \chi_{v}$ ). The figure illustrates the fact that there are 2 asymptotic limits for values of $d_{a}$, one in the continuum regime $\left(d_{c a}\right)$, and the other in the free-molecular regime $\left(d_{v a}\right)$. Between these two limiting values, in the transition regime, the ratio of $d_{a}$ to $d_{v e}$ transitions smoothly from one limit to the other.

and in laboratory-generated soot particles as described in the companion article (Slowik et al. 2004).

Effects of Flow Regime on Aerodynamic Measurements. As was stated above, for a given particle the value of $d_{a}$ changes with the flow regime (see Equations (28) and (29)). The dependence of aerodynamic diameter on flow regime is illustrated in Figure 3 , which is a plot of the ratio $d_{a} / d_{v e}$ as a function of $\mathrm{Kn}$. This figure is based on based on Equation (28), with the restriction/assumption that $\chi$ is the same in all flow regimes. Each curve represents a unique particle (i.e., $d_{v e}, \rho_{p}$, and $\chi$ do not change along a particular curve). Changes in the $d_{a} / d_{v e}$ ratio for each curve are due only to the changes in the flow regime where the particle aerodynamic diameter is determined. Figure 3 shows that an individual particle does not have a unique $d_{a}$. Rather, $d_{a}$ asymptotically approaches two unique values in the continuum regime $\left(d_{c a}\right)$ and the free-molecular regime $\left(d_{v a}\right)$, but takes on values between these limits in the transition regime. As shown in Figure $3, d_{c a}$ may be smaller or larger than $d_{v a}$ depending on the value of $\rho_{p} /\left(\rho_{0} \chi\right)$. $d_{v e}<d_{a}$ and $d_{v a}>d_{c a}$ when $\rho_{p} /\left(\rho_{0} \chi\right)>1$. $d_{v e}>d_{a}$ and $d_{v a}<d_{c a}$ when $\rho_{p} /\left(\rho_{0} \chi\right)<1$. In the following two sections, expressions are derived for the asymptotic limits of the aerodynamic diameter in the continuum regime $\left(d_{c a}\right)$ and the free-molecular regime $\left(d_{v a}\right)$.

Continuum Regime Aerodynamic Diameter $\left(d_{c a}\right)$. In the limit of the continuum regime, the aerodynamic diameter is denoted as $d_{c a}$. As stated above, in the continuum regime, $C_{c}\left(d_{c a}\right)=$ $C_{c}\left(d_{v e}\right)=1$. Using this relationship, Equation (28) can be expressed as

$$
d_{c a}=d_{v e} \sqrt{\frac{\rho_{p}}{\rho_{0} \chi_{c}}} .
$$

This diameter is measured by instruments such as an aerodynamic particle sizer (APS), which accelerates particles in a gas jet at ambient pressure and measures particle time of flight to size the (mostly supermicron) particles. (Ananth and Wilson 1988; Brockmann and Rader 1990). Some additional corrections apply in the APS for large particles with Reynolds number greater than 0.5 , because in this case the drag is non-Stokesian, i.e., it is not represented by Equation (11) (Wang and John 1987; Ananth and Wilson 1988; Tsai et al. 2004). Relative humidity variations during sizing, as well as deformations of liquid droplets as they are sized, may also change the particle size reported by the APS (McMurry 2000). It is possible that similar effects may exist in other aerodynamic sizing techniques.

Vacuum Aerodynamic Diameter $\left(d_{v a}\right)$. In the free-molecular regime, the aerodynamic diameter is called the vacuum aerodynamic diameter $\left(d_{v a}\right)$. Using Equation (13) for the slip correction in the free molecular regime in Equation (28), we can show that the vacuum aerodynamic diameter is related to $d_{v e}$ by (Jimenez et al. 2003a, b)

$$
d_{v a}=\frac{\rho_{p}}{\rho_{0}} \frac{d_{v e}}{\chi_{v}} .
$$

The error in this equation with respect to the exact equation (Equation (28), from using the simplification in Equation (13)) increases as $\chi_{v}$ increases, but for particles with $d_{v a}=1 \mu \mathrm{m}$, $\chi_{v}=3$, and standard density, the error is only $1.6 \%$ at a pressure of 1.5 Torr $(200 \mathrm{~Pa})$. Note that the calculation of $d_{v e}$ from a measurement of $d_{v a}$ requires knowledge of both the particle density and the dynamic shape factor.

The vacuum aerodynamic diameter $\left(d_{v a}\right)$ is now frequently measured in instruments that use low-pressure $(\sim 1.5$ Torr, $200 \mathrm{~Pa}$ ) aerodynamic lens systems as inlets, such as many aerosol mass spectrometers. In these devices, a series of axisymmetric lenses collimate aerosol particles into a tightly focused beam (Liu et al. 1995a, b; Zhang et al. 2002, 2004b). Several research groups have reported measurements of $d_{v a}$ (Zelenyuk et al. 1999; Jayne et al. 2000; Buzorius et al. 2002). In addition, the electrical low-pressure impactor (ELPI) measures $d_{v a}$ for smaller particles $\left(d_{v a}<33 \mathrm{~nm}\right)$. Other lens systems that size particles at higher pressures ( 200 Torr, $26.6 \mathrm{kPa}$ ) have also been designed and implemented (Schreiner et al. 2002; Cziczo et al. 2003). Most submicron particles are in the transition regime in these lenses.

Sizing with an aerodynamic lens is accomplished by measuring the size-dependent velocity that the particles acquire during the mild supersonic expansion into vacuum that occurs at the end of an aerodynamic lens. In the Liu et al. (1995a, b) design as implemented in the AMS this expansion is from approximately 1.5 Torr (200 Pa, $\lambda \sim 27.5 \mu \mathrm{m}, \mathrm{Kn} \sim 55$ for $1 \mu \mathrm{m})$ in the lens to about $2 \times 10^{-2}$ Torr (2.6 Pa, Kn $=4000$ for $1 \mu \mathrm{m}$ ) (Zhang et al. 2002, 2004b). In the AMS, about $1 \mathrm{~cm}$ after the end of the expansion the particles enter a differentially pumped high vacuum region $\left(10^{-5}\right.$ Torr, $0.001 \mathrm{~Pa} ; \mathrm{Kn}=8 \times 10^{6}$ for $1 \mu \mathrm{m}$ particles, and later $10^{-8}$ Torr or $10^{-6} \mathrm{~Pa}$ in the detection region). In this region the particle velocity remains constant due to the lack of 
collisions with gas molecules. The effect of gravity is negligible since it produces a vertical velocity of only $\sim 0.03 \mathrm{~m} \mathrm{~s}^{-1}$ compared to a horizontal velocity of $\sim 150 \mathrm{~m} \mathrm{~s}^{-1}$. In the particle time of flight, typically $\sim 3 \mathrm{~ms}$, the vertical displacement is only about $0.15 \mathrm{~mm}$, which is much smaller than the vaporizer radius of $1.65 \mathrm{~mm}$.

\section{The Jayne Shape Factor}

A different definition of a shape factor was proposed by Jayne et al. (2000) by comparing $d_{v a}$ to $d_{m}$ :

$$
S=\frac{d_{v a}}{d_{m}} \frac{\rho_{0}}{\rho_{m}} .
$$

$S$ has become known as the Jayne shape factor (Note, $\rho_{0}=$ $1.0 \mathrm{~g} \mathrm{~cm}^{-3}$ ). The advantage of $S$ is that it can be easily determined from tandem DMA-AMS measurements for particles of known $\rho_{m}$, where a DMA is used to select particles with a given $d_{m}$ and the AMS is used to measure their $d_{v a}$. Notice that $S$ is not the reciprocal of $\chi_{v}$, as was erroneously stated in Jimenez et al. (2003a) and corrected in Jimenez et al. (2003b). It can be shown using Equations (25) and (31) that the relationship between $S$ and $\chi$ is given by

$$
S=\frac{\rho_{p}}{\rho_{m}} \frac{C_{c}\left(d_{v e}\right)}{\chi_{t} \cdot \chi_{v} \cdot C_{c}\left(d_{m}\right)}=\frac{C_{c}\left(d_{v e}\right)}{\delta^{3} \chi_{t} \cdot \chi_{v} \cdot C_{c}\left(d_{m}\right)},
$$

where the slip correction factors are calculated at the DMA pressure. When the particles are mildly nonspherical, the ratio of the slip correction factors $\left(C_{c}\left(d_{v e}\right) / C_{c}\left(d_{m}\right)\right)$ near the continuum regime limit is $\sim 1$ and near the free molecular regime limit $\sim \chi_{t}^{1 / 2}$. For particles in the transition regime there will be a smooth transition between those values. Thus the Jayne shape factor can be approximated towards the continuum regime limit for particles in the DMA as

$$
S \cong \frac{1}{\delta^{3} \chi_{v} \chi_{t}}
$$

and towards the free molecular limit in the DMA as

$$
S \cong \frac{1}{\delta^{3} \chi_{v} \chi_{t}^{1 / 2}}
$$

If the particle does not have internal voids (i.e., $\delta=1$ ), then $S$ will be $\sim 1 / \chi^{2}$ in the continuum limit to $\sim 1 / \chi^{3 / 2}$ in the freemolecular limit. This is a useful estimate for relating the Jayne shape factor to the dynamic shape factor for particles that are mildly nonspherical.

\section{PREVIOUS STUDIES OF PARTICLE MORPHOLOGY}

\section{Particle Shape}

Early studies of shape and density effects in aerosol sizing used aerosol centrifuges and transmission electron microscopy (TEM) in addition to theoretical calculations. The effect of shape on sizing was investigated and the dynamic shape factor for aggregate particles was determined with this technique (Stober 1972; Allen et al. 1979; Kasper 1982a, b). Mobility classification with DMAs followed by TEM analysis has been used to test the theoretical knowledge of shape effects and to study the shape and sizing of agglomerate particles (Rogak et al. 1993). Brockmann and Rader (1990) used an APS and a cascade impactor in parallel to experimentally determine the dynamic shape factor of Bermuda grass spores, cerium oxide particles, and iron-alumina particles. More recently, Park et al. (2003, 2004a, b) used tandem DMA and aerosol particle mass analyzer (APM), and tandem DMA-TEM measurements to determine the dynamic shape factors and density of diesel soot as a function of mobility diameter. Theoretical calculations of drag and shape factors have also been performed for particles in the free molecular regime (Dahneke 1973a, b, c; Cheng 1991; Baron et al. 2001b); however, until recently there has been little experimental data to test the calculations.

\section{Particle Density}

Numerous methods of measuring particle density have been discussed in the literature for both laboratory and field studies (Emets et al. 1992; Schleicher et al. 1995; Ehara and Shin 1998; Le Bronec et al. 1999; Morawska et al. 1999; McMurry et al. 2002; Pitz et al. 2003). For nonspherical particles, studies generally rely on the assumption of spherical particles with a physical diameter equal to the mobility diameter for volume and density calculations. When particles are not spherical this technique yields an "effective" or "apparent" density, not necessarily a true measure of particle density. The precise definition of such an "effective" density varies with the measurement technique (see section "Effective Density" below). Emets et al. (1992) and Le Bronec et al. (1999) used the effect of gravity on mobility transfer functions to determine particle mass. Assuming spherical particles and estimating particle volume using the measured mobility diameter yielded a calculation of particle density with $10 \%$ error (Emets et al. 1992) and less than 5\% error (Le Bronec et al. 1999) for polystyrene latex spheres.

Kelly and McMurry (1992) and Schleicher et al. (1995) combined DMA and impactor measurements to determine a relationship between mass and mobility for various particle types, including aggregates. Assuming spherical particles, they calculated effective density based on the measured mass from the impactor. For aggregates their calculated effective density was much lower than the bulk density of the particle material. Ehara and Shin (1998) developed an APM, which balances centrifugal force and electrostatic force to determine particle mass/charge. A tandem DMA-APM experiment allows the determination of particle mass and electrical mobility of aerosol particles and subsequent calculation of effective density. McMurry et al. (2002) used the tandem DMA-APM setup to measure an effective density of ambient aerosol particles in Atlanta, GA. Results indicated the presence of both a spherical component and a nonspherical component in the ambient aerosol. The nonspherical 
component was hypothesized to be chain agglomerates of soot. Morawska et al. (1999) and Pitz et al. (2003) determined $\mathrm{PM}_{2.5}$ mass using a tapered element oscillating microbalance (TEOM) and used integrated number distributions from an SMPS and laser aerosol spectrometer to determine bulk ambient particle apparent density. In both studies a wide range of apparent densities were reported, from less than $1 \mathrm{~g} \mathrm{~cm}^{-3}$ to greater than $3 \mathrm{~g} \mathrm{~cm}^{-3}$. Khlystov et al. (2004) used a similar technique in Pittsburgh using an APS instead of the laser aerosol spectrometer. Estimates of total $\mathrm{PM}_{2.5}$ using size integrated distributions and an assumed density indicate an error of $\pm 20 \%$ when compared to TEOM measurements, likely due to both changes in aerosol composition (density change) and aerosol shape. These authors also note that the technique was specific for Pittsburgh and that aerosol characteristics of shape and density are likely different elsewhere.

\section{Mobility and Vacuum Aerodynamic Diameter}

On-line instruments that can measure the mobility diameter of submicron particles have been available for some time (Flagan 2001). The development of aerodynamic lenses (Liu et al. 1995a, b; Zhang et al. 2002, 2004b) has lead to the widespread online measurement of the aerodynamic diameter of submicron particles in the free-molecular regime, also known as vacuum aerodynamic diameter (Jimenez et al. 2003a, b). The Aerodyne AMS (Jayne et al. 2000) is one such instrument. In this article we will refer to the AMS for simplicity, but all results also apply to other instrument designs that use the same type of an inlet. The development of an ELPI also allows the measurement of aerodynamic diameter of particles over different regimes for different particle sizes, including the free-molecular regime for particles $\sim 33 \mathrm{~nm}$ and smaller (Maricq et al. 2000; Van Gulijk et al. 2004; Virtanen et al. 2004).

\section{INFORMATION FROM $d_{m}$ AND $d_{v a}$ MEASUREMENTS}

Since parallel or serial measurements of $d_{m}$ and $d_{v a}$ are easily obtainable, it is of interest to explore the information that can be obtained by performing both measurements for a given particle population. In tandem (serial) DMA-AMS measurements particles are first selected by mobility, and then sized aerodynamically. In performing this type of study, it is important to dry the particles before sizing them with the DMA, so that changes in water content due to evaporation in the AMS lens do not change the particles in between the $d_{m}$ and $d_{v a}$ measurements (see Appendix B).

\section{Density and Shape Factor}

By combining Equations (25) and (31) it can be shown that

$$
\frac{\frac{d_{v a} \chi_{v} \chi_{t} \rho_{0}}{\rho_{p}}}{C_{c}\left(\frac{d_{v a} \chi_{v} \rho_{0}}{\rho_{p}}\right)}=\frac{d_{m}}{C_{c}\left(d_{m}\right)} .
$$

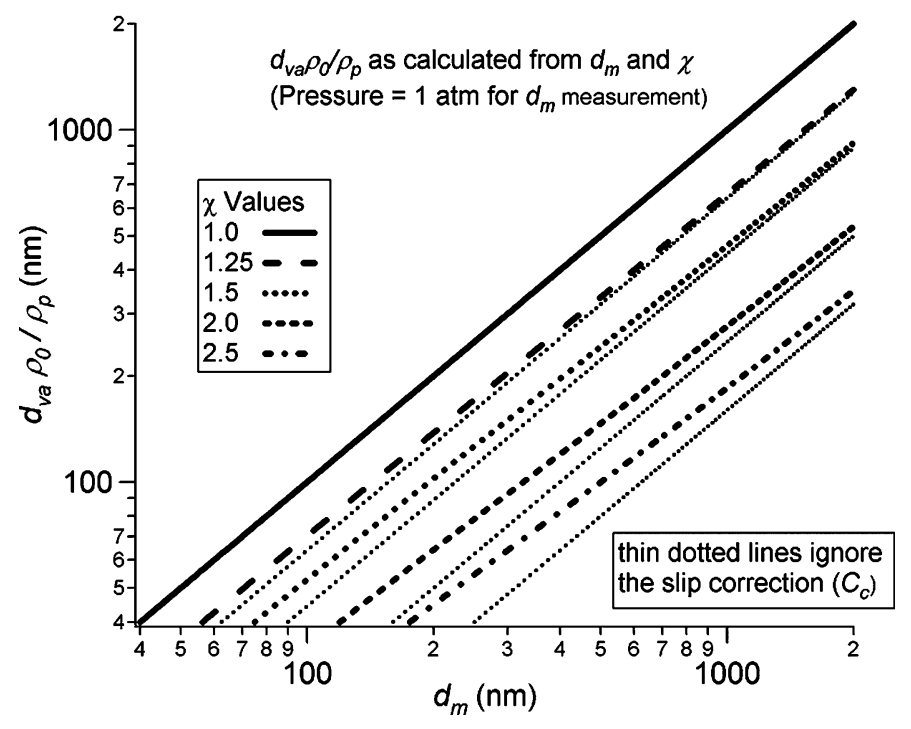

Figure 4. Relationship between $d_{v a} \rho_{0} / \rho_{p}$ and $d_{m}$ as a function of $\chi$ (with the assumption $\chi_{t} \sim \chi_{v}$ ). Given measurements of $d_{m}$ and $d_{v a}$ and an estimate of $\rho_{p}$, the figure can be used to estimate $\chi$. Note that all values of $d_{m}$ in this paper are calculated assuming 1 atm pressure.

Equation (36) is the general expression for relating $d_{v a}$ and $d_{m}$ measurements. Jimenez et al. (2003a) showed that for particles with a constant $d_{v e}$ and standard density, $d_{m}$ and $d_{v a}$ change in opposite directions as $\chi_{t}(\mathrm{Kn})$ and $\chi_{v}$ increase. In a similar fashion, we show the relationship between $d_{v a} \rho_{0} / \rho_{p}$ and $d_{m}$ in Figure 4, using Equation (36), for several values of $\chi$ with the assumption $\chi_{t} \approx \chi_{v}$. Note that $\rho_{p}$ is the particle density defined in Equation (5), which in the absence of particle void spaces equals the material density $\left(\rho_{m}\right)$. Figure 4 is to be used as a "map" to estimate a $\chi$ value from measurements of $d_{v a}$ and $d_{m}$, if $\rho_{p}$ is known or can be reasonably estimated. The dotted lines in Figure 4 illustrate the effect of slip correction on this calculation. If the slip correction is ignored in Equation (36) then the thin dotted lines are obtained. As expected, the difference in the slip correction factors is less important for large particles, where flow around the particle in the DMA is closer to the continuum regime.

In a situation when only $d_{m}$ and $d_{v a}$ can be determined, but no other information on shape or density is available, there is a considerable range of values for $\chi$ and $\rho_{p}$ that yield the same $d_{m}$ and $d_{v a}$ measurements. These ranges can be estimated from Figure 5. Figure 5 illustrates the range and combinations of $\rho_{p}$ and $\chi$ that will solve Equation (36) for a given pair of $d_{m}$ and $d_{v a}$ measurements (again assuming $\chi_{t} \approx \chi_{v}$ ). For example, if $d_{v a}=$ $100 \mathrm{~nm}$ and $d_{m}=200 \mathrm{~nm}$, then the possible $\rho_{p}$ and $\chi$ values are linked, ranging from $\rho_{p}=0.5 \mathrm{~g} \mathrm{~cm}^{-3}$ and $\chi=1$, to $\rho_{p}=$ $3 \mathrm{~g} \mathrm{~cm}^{-3}$ and $\chi=3$ (and also higher values of both parameters). The minimum particle density can be estimated by setting $\chi=1$.

\section{Calculations of $\rho_{p}$ and $\chi$ for Specific Particle Types}

The simultaneous determination of $d_{m}$ and $d_{v a}$, together with Equation (36) provides the basis for bringing together other 

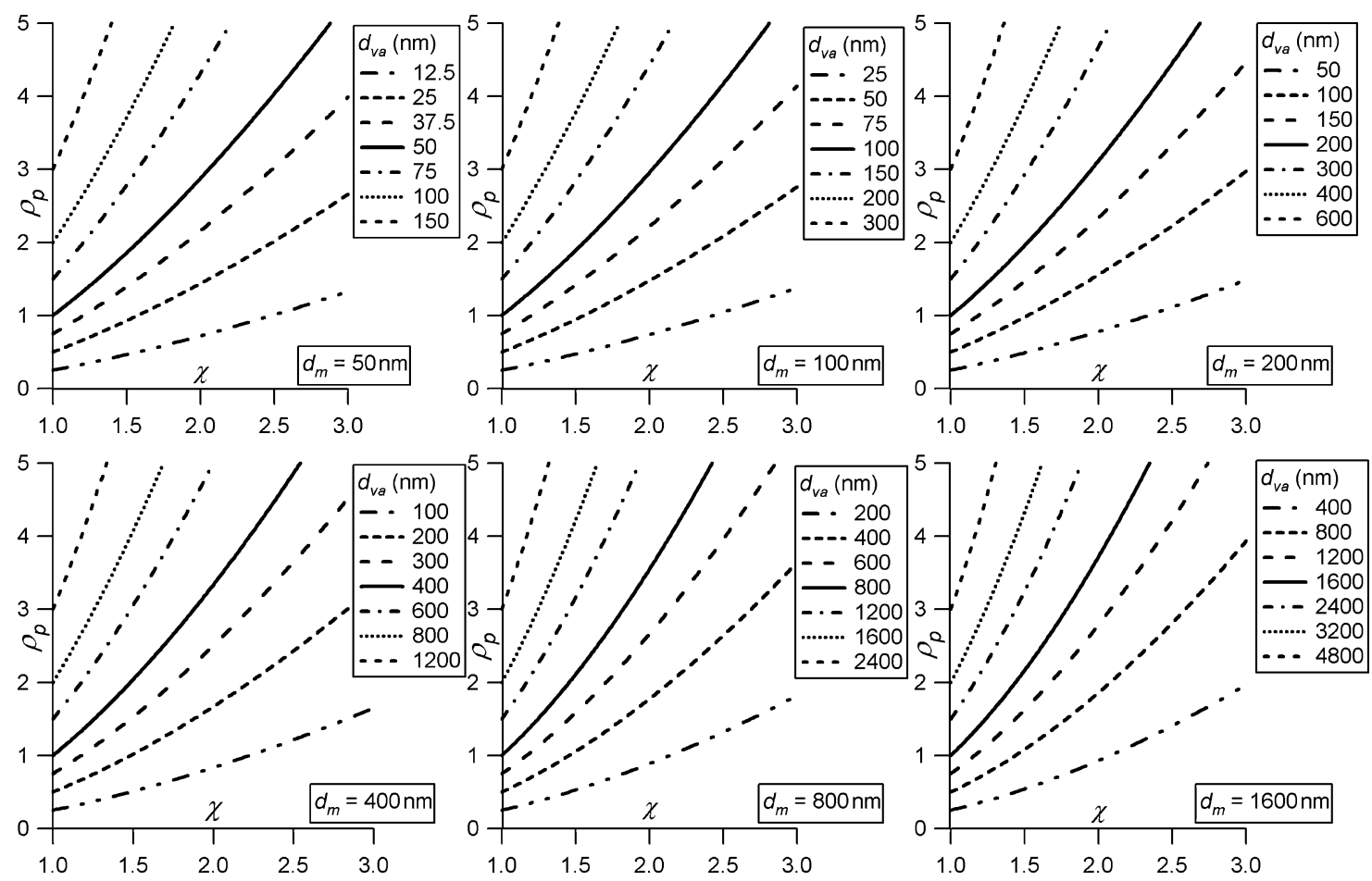

Figure 5. This figure shows the combinations of $\chi$ and $\rho_{p}$ that are possible given various combinations of $d_{m}$ and $d_{v a}$ measurements with the assumption $\chi_{c} \approx \chi_{t} \approx \chi_{v}$ ). Each plot represents a particular value of $d_{m}$, while the individual traces in the plot represent particular values of $d_{v a}$.

measurements and assumptions about the particles in order to self-consistently estimate important particle properties such as density, volume, and mass. Table 1 applies the framework presented here to different types of particles. The particles are classified by their external and internal morphologies. The table progresses from simple to more complex particle types. Valid relations between parameters for each particle type are also listed.

Spherical Particles. In the simplest case of spherical particles (type A in Table 1), particle volume can be directly calculated because $d_{v e}=d_{m}$. Particle density, $\rho_{p}$, is then determined for type A particles from the measurements of $d_{m}$ and $d_{v a}$ via Equation (36), which simplifies to

$$
\rho_{p}=\frac{d_{v a}}{d_{m}} \rho_{0} .
$$

For a spherical particle consisting of multiple solid or liquid phases, this analysis applies and $\rho_{p}$ is then the average particle density as determined by Equation (5).

The above analysis is also applicable for spherical particles with internal void spaces (type B in Table 1). Note that particle density will not be equal to material density for type B due to the effect of the voids. If the material density, $\rho_{m}$, is known, the void volume fraction of the particle $(\omega)$ and the particle volume can be calculated with Equations (6-8).

Compact Aggregates. Particle volume can also be estimated for compact aggregates (type $\mathrm{C}$ particles) solely from measure- ments of $d_{v a}$ and $d_{m}$, but this requires the assumption of a spherical shape. This has been shown to be a reasonable approximation for some real aggregate particle types (Stober 1972; Kasper 1982a). All relationships are the same as for type B once we assume sphericity (i.e., $d_{v e} \approx d_{m}$ ). Without the assumption of sphericity, the particles can be treated by the methods described in the next section for irregular and aggregate particles.

Irregular Particles. For irregular particles (types D, E, F, and $\mathrm{G}$ in Table 1), the spherical assumption is not a good approximation. Fractal aggregates are a special subset of irregular particles and are discussed in more detail below. For irregular particles neither $\chi_{t}(\mathrm{Kn})$ nor $\chi_{v}$ are equal to 1 , and therefore there are 2 additional unknowns in Equation (36). Two additional linearly independent measurements or approximations are needed to solve the system and determine $d_{v e}, \rho_{p}, \chi_{t}$, and $\chi_{v}$. The void volume fraction $(\omega)$ can then be determined if the material density $\left(\rho_{m}\right)$ is known from the chemical composition.

Knowledge of particle composition can provide one additional constraint on the system by providing an estimate of $\rho_{p}$. If the additional assumption of no internal voids is made (i.e., $\delta=1$ ), then $\rho_{p}=\rho_{m}$. In many laboratory experiments, the particle composition and material density are known because the particle generation system is well defined.

A measurement of total particle mass (e.g., with an APM) can provide a second independent measurement and constrain the system via Equation (5). If the particles are known to consist 
Table 1

Summary of different particle types and associated relations of particle density, material density, and shape factor

\begin{tabular}{|c|c|c|c|c|}
\hline Particle type & & Diameter relations & $\rho_{p}$ and $\rho_{m}$ relations & $\chi$ and $\chi^{\prime}$ relations \\
\hline A & $\begin{array}{l}\text { Sphere } \\
\text { (no voids) }\end{array}$ & $\begin{array}{l}d_{m e}=d_{v e} \\
d_{m}=d_{v e} \\
\frac{d_{v a} \rho_{0}}{\rho_{p}}=d_{v e}\end{array}$ & $\rho_{p}=\rho_{m}$ & $\chi=\chi^{\prime}=1$ \\
\hline B & $\begin{array}{l}\text { Sphere } \\
\text { (internal } \\
\text { voids) }\end{array}$ & $\begin{array}{l}d_{m e}=d_{m e} * \delta \\
d_{m}=d_{v e} \\
\frac{d_{v a} \rho_{0}}{\rho_{p}}=d_{v e}\end{array}$ & $\begin{array}{l}\rho_{p}<\rho_{m} \\
\delta^{3} \rho_{p}=\rho_{m}\end{array}$ & $\begin{array}{l}\chi=1 \\
\chi^{\prime}=\delta \frac{C c\left(d_{m e}\right)}{C c\left(\delta \cdot d_{m e}\right)}\end{array}$ \\
\hline $\mathrm{C}$ & $\begin{array}{l}\text { ompact } \\
\text { ggregate } \\
\text { nternal voids) }\end{array}$ & $\begin{array}{l}d_{m e}=d_{m e} * \delta \\
\text { Assume: } \\
d_{m} \approx d_{v e}\end{array}$ & $\begin{array}{l}\rho_{p}<\rho_{m} \\
\delta^{3} \rho_{p}=\rho_{m}\end{array}$ & $\begin{array}{l}\chi \approx 1 \\
\chi^{\prime}=\delta \frac{C c\left(d_{m e}\right)}{C c\left(\delta \cdot d_{m e}\right)}\end{array}$ \\
\hline $\mathrm{D}$ & $\begin{array}{l}\text { Irregular } \\
\text { (no voids) }\end{array}$ & & & \\
\hline & & $\begin{array}{l}d_{m e}=d_{v e} \\
d_{m}>d_{v e}\end{array}$ & $\rho_{p}=\rho_{m}$ & $\chi^{\prime}=\chi>1$ \\
\hline $\mathrm{E}$ & $\begin{array}{l}\text { Aggregate } \\
\text { (no voids) }\end{array}$ & & & \\
\hline $\mathrm{F}$ & $\begin{array}{l}\text { Irregular } \\
\text { (internal } \\
\text { voids) }\end{array}$ & & & \\
\hline G & $\begin{array}{l}\text { Aggregate } \\
\text { (internal } \\
\text { voids) }\end{array}$ & $\begin{array}{l}d_{m e}=d_{m e} * \delta \\
d_{m}>d_{v e}\end{array}$ & $\begin{array}{l}\rho_{p}<\rho_{m} \\
\delta^{3} \rho_{p}=\rho_{m}\end{array}$ & $\begin{array}{l}\chi^{\prime}>\chi>1 \\
\chi^{\prime}=\chi \cdot \delta \frac{C c\left(d_{m e}\right)}{C c\left(\delta \cdot d_{m e}\right)}\end{array}$ \\
\hline
\end{tabular}

Reasonable assumptions about particle properties are also included.

Aggregate particles are a special case of irregular particles.

of nonrefractory components, then quantitative measurements of particle mass can be obtained using the AMS, also adding a constraint to the system. Other methods are also available for quantitatively measuring particle composition or mass.

An approximation that can be made to reduce the underdetermination of the system is to assume $\chi_{t}(\mathrm{Kn}) \approx \chi_{v}$. Although it is known that $\chi$ can change with flow regime, this assumption provides a starting point for the application of the theory in the absence of additional information. With this assumption the number of unknowns is reduced by 1 , therefore only one additional independent measurement or assumption is needed to solve the system of equations. With the assumption $\chi_{t}(\mathrm{Kn}) \approx \chi_{v}$, 
Equation (36) still provides only a relationship between $\rho_{p}$ and $\chi$ (such as is illustrated in Figures 4 and 5). As is shown below, an additional constraint can be derived for fractal aggregates (see below). Alternatively, if in addition to the assumption that $\chi_{t}(\mathrm{Kn}) \approx \chi_{v}$ particle density can be estimated either from measurement or knowledge of particle material, then Equation (36) can be solved for $\chi$. Thus $d_{v e}$ can then be estimated from Equation (31), and $m_{p}$ and $V_{p}$ can be estimated as described in section "Volume and Density." An application of this approach is presented in the companion article (Slowik et al. 2004).

\section{Effective Density}

Effective density (or apparent density) is a parameter often defined in the literature from a combination of two aerosol measurements (Baron et al. 2001b; Hand et al. 2002; McMurry et al. 2002; Jimenez et al. 2003a). Various definitions of effective density are used, and different definitions may yield different values for a given particle. Comparing calculated effective densities from various measurements can be misleading if the densities are not derived in a consistent manner. Thus, it is important to understand how a particular effective density is derived and what its proper uses are. In this section we review and interpret four commonly used literature definitions of effective density within the analytical framework presented in this article.

Particle Density $\left(\rho_{p}\right)$. The particle density $\left(\rho_{p}\right)$ as defined in Equation (5) may be considered an effective density when compared to bulk material density $\left(\rho_{m}\right)$ of the particle in question (Baron et al. 2001b). In this case $\rho_{p}$ is different from the material density $\rho_{m}$ only when the particle contains internal void spaces. The difference between $\rho_{m}$ and $\rho_{p}$ is then purely a function of the volume fraction of internal void spaces in the particle $(\omega)$, see Equations (3-9).

Effective Density from Mobility and Mass Measurements $\left(\rho_{\text {eff }}^{I}\right)$. A common definition of effective density $\left(\rho_{\text {eff }}^{I}\right)$ is the ratio of the measured particle mass $\left(m_{p}\right)$ to the particle volume calculated assuming a spherical particle with a diameter equal to the measured $d_{m}$. The volume defined with this assumption is sometimes referred to as the apparent volume $\left(V_{a}\right)$. The required parameters, $m_{p}$ and $d_{m}$, are readily obtained from a mass measurement in addition to a mobility measurement with a DMA/SMPS system. For example, the tandem DMA-APM setup as reported by McMurry et al. (2002) can be used to perform this measurement. A DMA selects particles of a certain electrical mobility followed by downstream scanning of mass with an APM. A parallel SMPS-TEOM system can also be used to determine this effective density for the particle population rather than for individual particles (Morawska et al. 1999; Pitz et al. 2003). By definition, the particle mass can be written in terms of $\rho_{\text {eff }}^{I}$ as

$$
m_{p}=\rho_{\text {eff }}^{I} \cdot \frac{\pi}{6} d_{m}^{3}=\rho_{e f f}^{I} \cdot V_{a} .
$$

Substituting for $m_{p}$ (see Equation (5)), Equation (38) can be rewritten as

$$
\frac{\pi}{6} \cdot d_{v e}{ }^{3} \rho_{p}=\frac{\pi}{6} \cdot d_{m}^{3} \rho_{e f f}^{I}
$$

Simplifying Equation (39), $\rho_{\text {eff }}^{I}$ can be expressed as

$$
\rho_{\text {eff }}^{I}=\rho_{p}\left(\frac{d_{v e}}{d_{m}}\right)^{3} .
$$

This effective density $\left(\rho_{\text {eff }}^{I}\right)$ is the particle density that a sphere with diameter $d_{m}$ would need to have the same mass as the actual particle. For spheres $\rho_{\text {eff }}^{I}=\rho_{p}$. Since for irregular particles an SMPS yields diameters larger than their volume equivalent diameter, $\rho_{\text {eff }}^{I} \leq \rho_{p}$. Conceptually, the definition of $\rho_{\text {eff }}^{I}$ uses the difference between $d_{m}$ and $d_{v e}$ to estimate an external pseudovoid fraction $\left(\omega^{\prime}\right)$ of the particle volume referenced to $d_{m}: \omega^{\prime}=\left(1-\left(d_{v e} / d_{m}\right)^{3}\right)$. The external pseudovoid fraction is the fraction of unoccupied volume existing between the envelope of the particle material and a spherical envelope of diameter $d_{m}$ (see, e.g., Figure 5 in Van Gulijk et al. 2004). Note that this external pseudovoid fraction is completely due to external physical morphology and is unrelated to the internal void fraction $\left(\omega=1-1 / \delta^{3}\right.$, defined above).

By combining Equations (39) and (31), $\rho_{\text {eff }}^{I}$ can be expressed in terms of parameters related to DMA and AMS measurements $\left(d_{m}, d_{v a}, \rho_{p}\right.$, and $\left.\chi_{v}\right)$ as

$$
\frac{\pi}{6} \cdot\left(\frac{d_{v a} \cdot \chi_{v} \rho_{0}}{\rho_{p}}\right)^{3} \rho_{p}=\frac{\pi}{6} \cdot d_{m}{ }^{3} \rho_{e f f}^{I},
$$

or

$$
\rho_{e f f}^{I}=\rho_{0}\left(\frac{d_{v a} \cdot \chi_{v}}{d_{m}}\right)^{3}\left(\frac{\rho_{0}}{\rho_{p}}\right)^{2}
$$

Effective Density as a Fitted Parameter $\left(\rho_{\text {eff }}^{I I}\right)$. Another definition of effective density $\left(\rho_{e f f}^{I I}\right)$ is given by Hand et al. (2002) and also used by Khlystov et al. (2004). Hand et al. (2002) combined size distribution data from a DMA, an optical particle counter (OPC), and an APS, and used overlap regions to estimate refractive index and (a third definition of) effective density:

$$
\rho_{\text {eff }}^{I I}=\frac{\rho_{p}}{\chi} .
$$

Note that this definition is different from that of McMurry et al. (2002) $\left(\rho_{e f f}^{I}\right)$. In addition, the above equation requires a determination of $\rho_{p}$ and $\chi$. In the above studies, $\rho_{\text {eff }}^{I I}$ was a fitted parameter in the analysis algorithm, since neither the particle density nor the dynamic shape factor were measured by the instrumentation. For spheres, $\rho_{e f f}^{I I}=\rho_{p}$ and for nonspherical particles $\rho_{e f f}^{I I}<\rho_{p}$. 
Effective Density from Mobility and Aerodynamic Measurements $\left(\rho_{\text {eff }}^{I I I}\right)$. An alternative estimation of effective density can be performed by comparing mobility and aerodynamic diameter measurements (e.g., Kelly and McMurry 1992; Stein et al. 1994). The specific definition when $d_{v a}$ is measured is presented by Jimenez et al. (2003a) as

$$
\rho_{\text {eff }}^{I I I}=\frac{d_{v a}}{d_{m}} \rho_{0} .
$$

Using Equations (32), (33), and (44), it can be shown that

$$
\rho_{e f f}^{I I I}=\rho_{m} \cdot S=\rho_{p} \frac{C_{c}\left(d_{v e}\right)}{\chi_{t} \cdot \chi_{v} \cdot C_{c}\left(d_{m}\right)} .
$$

Again, this definition of effective density is different from the three given above. The parameters required to calculate $\rho_{\text {eff }}^{I I I}$ can be easily obtained experimentally with a tandem DMA-AMS system. Note that for spheres $\rho_{\text {eff }}^{I I I}=\rho_{p}$, and for nonspherical particles $\rho_{\text {eff }}^{I I I}<\rho_{p}$.

Figure 6 shows a numerical example of the effective density calculated via the latter three definitions as a function of $\chi$. For $\rho_{\text {eff }}^{I I I}$, it was assumed that $\chi_{t} \approx \chi_{v}$. All of the definitions successfully demonstrate that the more irregular the particle, the lower the effective density. However, as is evident, the definitions do not yield the same numerical values because they capture slightly different particle properties.

\section{Mass of Irregular Particles Estimated from DMA-AMS Measurements}

The mass of an irregular particle is difficult to estimate from typical aerosol measurements. An APM is able to measure the

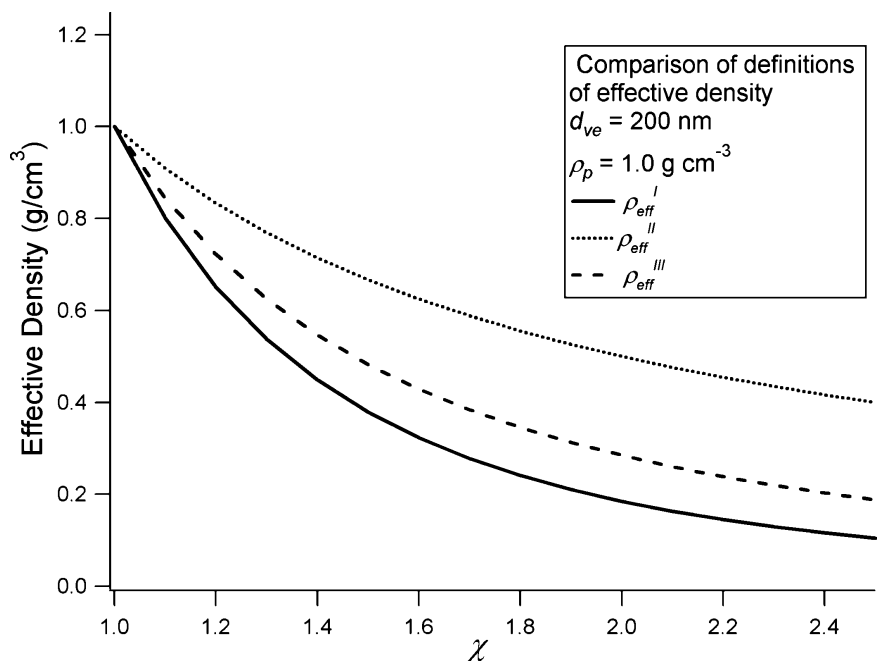

Figure 6. Comparison of different definitions of effective density as a function of $\chi$ for a particle with $d_{v e}=200 \mathrm{~nm}$ and $\rho_{p}=1.0 \mathrm{~g} \mathrm{~cm}^{-3}$, and assuming $\chi_{t} \approx \chi_{v}$. For each definition, the effective density decreases with increasing $\chi$, however the numerical values of the effective densities are significantly different. mass of individual particles directly; however, very few of these instruments are available, and consequently their use is not widespread. Other techniques would be useful to estimate the mass of irregular particles. A $d_{m}$ measurement combined with an effective density estimate $\left(\rho_{\text {eff }}^{I}\right)$ can be used to estimate particle mass using Equation (38). However, $\rho_{\text {eff }}^{I}$ can only be determined if particle mass is already known. Figure 6 shows that $\rho_{\text {eff }}^{I}$ is not very different from $\rho_{\text {eff }}^{I I I}$, which can be calculated from only $d_{m}$ and $d_{v a}$ measurements. In this section we estimate the error in the particle mass estimate if the measured $\rho_{\text {eff }}^{\text {III }}$ is used to replace $\rho_{\text {eff }}^{I}$ in Equation (38). The usefulness of this approach is that particle mass can be estimated from two readily available measurements $\left(d_{m}\right.$ and $\left.d_{v a}\right)$, without any information about $\rho_{p}$ or $\chi$. The comparison of the two methods requires the assumption $\chi_{t}(\mathrm{Kn}) \approx \chi_{v}$, which introduces some uncertainty into the comparison.

To make this comparison we begin with the exact calculation of $m_{p}$, which can be obtained with knowledge of only $d_{v e}$ and $\rho_{p}$ via Equation (5). Using the same $d_{v e}$ and for any $\chi, d_{m}$ can be calculated using Equation (25). Making the additional assumption $\chi_{t} \approx \chi_{v}$, we can calculate $d_{v a}$. We can then calculate an estimated $m_{p}$ by replacing $\rho_{\text {eff }}^{I}$ by $\rho_{\text {eff }}^{I I I}$ in Equation (38), where $\rho_{\text {eff }}^{I I I}$ is found using the above calculations of $d_{v a}$ and $d_{m}$. Figure 7 shows the ratio of the estimated mass via $\rho_{\text {eff }}^{I I I}$ to the exact mass, as calculated with $d_{v e}$ and $\rho_{p}$, as a function of $d_{m}$ and $\chi$. Note that the estimated error does not depend on $\rho_{p}$ because this dependence cancels out. For nonspherical particles the estimated mass is always larger than the actual mass. The estimated error increases with both $d_{m}$ and $\chi$. For small $\left(d_{m}<70 \mathrm{~nm}\right)$ or mildly irregular $(\chi<1.2)$ particles, the estimated error due to using the approximate formula is remarkably small $(<10 \%)$. For large or highly irregular particles, this approximation allows the

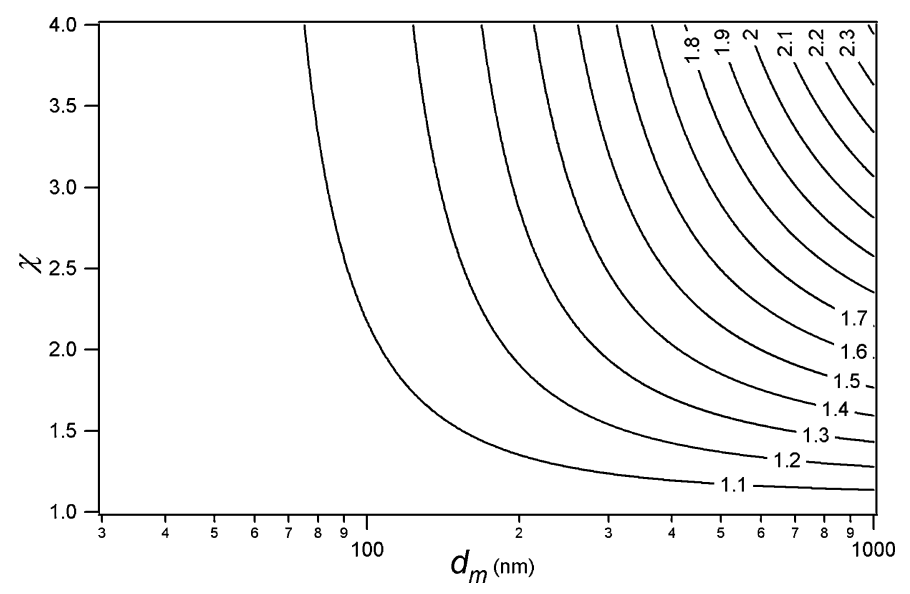

Figure 7. Contour plot of the ratio of the exact mass of a particle to the estimated mass of a particle replacing $\rho_{\text {eff }}^{I I I}$ with $\rho_{\text {eff }}^{I}$ in Equation (30) and assuming $\chi_{t} \approx \chi_{v}$. The error in the estimate increases as $d_{m}$ and $\chi$ increase, but it is remarkably small for mildly irregular particles and for small particles. 
estimation of particle mass from only $d_{m}$ and $d_{v a}$ measurements within about a factor of two, without any knowledge of $\rho_{p}$ or $\chi$.

Note that although the AMS can only measure the nonrefractory mass in the particles due to its thermal vaporation at $600^{\circ} \mathrm{C}$, the approximation presented here is also valid for particles with refractory components that cannot be measured by the AMS, or for components whose relative ionization efficiency is not known, since only a size measurement of the particle is needed.

A further implication of this result is that the mass of the $\mathrm{NH}_{4} \mathrm{NO}_{3}$ particles used on the ionization efficiency (IE) calibration of the AMS (Jimenez et al. 2003c) may be slightly overestimated, leading to an underestimation of $I E$. This is because the AMS data acquisition software (version 4.5 and earlier) estimates the mass of the calibration particles as $\rho_{\text {eff }}^{I I I} \cdot V_{a}$, which as we have shown in this section leads to a systematic overestimation of the particle mass. Fortunately, the estimated error for the typical AMS calibration particles $\left(d_{m}=350 \mathrm{~nm}\right.$ and $\left.S=0.8\right)$ is relatively small $(\sim 6.7 \%)$. Further research in this area is needed to increase to absolute accuracy of the mass concentrations reported by the AMS.

\section{FRACTAL AGGREGATES}

Aggregate particles are produced in a variety of processes such as combustion, pharmaceutical drug delivery, and in the manufacture of carbon black or other materials (Friedlander and Pui 2004). Typically these aggregates are composed of similarly sized primary particles (spherules) attached together. There are two common types of aggregate particles described in the literature.

The first type is a compact aggregate, whose external envelope is not far from spherical shape (i.e., type $\mathrm{C}$ on Table 1). In this case $\rho_{p}$ is a function of the packing density of the spherules (e.g., Stober 1972; Kasper 1982a). Calculations for type C particles were addressed above. We will focus here on a second type of aggregate particles for which the external envelope is highly nonspherical. Such particles are often termed fractal (Types E and $\mathrm{G}$ in Table 1) and have been studied extensively, both theoretically and experimentally (e.g., Stober 1972; Baron et al. 2001b). Combustion emissions such as diesel soot are a large source of fractal particles in the environment. These particles are of particular interest since they are highly irregular in nature, with dynamic shape factors often exceeding 2 (Stober 1972; Park et al. 2004a).

\section{Fractal Dimension}

Fractal dimension $\left(D_{f}\right)$ is a parameter that is often used to describe aggregate particles. The parameter, $D_{f}$, is defined from a relationship between the number of primary particles in an aggregate to a characteristic radius, $R$, typically the radius of gyration, by the following power law (Friedlander 2000)

$$
N_{p p} \sim R^{D_{f}} .
$$

Here $N_{p p}$ is the number of primary particles in the aggregate.
The fractal dimension can vary between 1 and 3 . For spheres $D_{f}=3$, compact agglomerates $D_{f} \approx 3$, and in the limit of infinitely long straight chain agglomerates $D_{f} \rightarrow 1$. A range of intermediate values have been reported both for laboratorygenerated combustion aerosol and also for ambient aerosol (Koylu and Faeth 1992; Katrinak et al. 1993; Park et al. 2003, 2004a). The fractal dimension can be estimated via the massmobility relationship (Park et al. 2003) based on the scaling laws developed by Schmidt-Ott et al. (1990). In the mass-mobility relationship, it is assumed that the number of the primary particles is proportional to $m_{p}$, which requires the primary particle size distribution to be constant for all values of $N_{p p}$ as well as the assumption that the primary particle density is constant (which may not be strictly true if the aggregate is coated by a second species). The mass-mobility relationship is expressed as

$$
m_{p}=C^{\prime}\left(\frac{d_{m}}{d_{p p}}\right)^{D_{f}}
$$

Here $C^{\prime}$ is a constant and $d_{p p}$ is the diameter of the spherules comprising the aggregate. Only the assumption of a constant primary particle size distribution is required to state that $V_{p}$ is directly proportional to $N_{p p}$. Equation (47) can then be rewritten as

$$
d_{v e}^{3}=C^{\prime \prime}\left(\frac{d_{m}}{d_{p p}}\right)^{D_{f}}
$$

Since $d_{m}$ can be measured and $d_{v e}$ and can be estimated as a function of $N_{p p}$ (for a given $d_{p p}$ ) as described above, then Equation (48) can be used to estimate a fractal dimension based on the mass-mobility relationship with known values of $d_{p p}$ and $N_{p p}$.

\section{Additional Constraint on Mass Calculation}

Rogak et al. (1993) applied results of numerical calculations from Dahneke (1973a, b, c) to fractal aggregates. Using these calculations, they showed that $d_{m}$ is approximately equal to the projected area diameter $\left(d_{A}\right)$ for fractal particles with a $D_{f}$ of 2.0 or higher and a primary particle size $\left(d_{p p}\right)$ of $\sim 33 \mathrm{~nm}$, well into the transition regime (up to $\mathrm{Kn}=0.3$ based on $\lambda=65 \mathrm{~nm}$ ). The projected area diameter is the diameter of a circle with the same area as the particle silhouette (Hinds 1999). It is expected that $d_{m}=d_{A}$ for the free-molecular regime, but this relationship breaks down in continuum regime flow. The fact that this relationship is conserved into the transition regime indicates that $d_{m}$ is a conserved quantity (since the projected area of a particle is conserved) for the range in which $d_{m}=d_{A}$. In this range we can rewrite Equation (25) for $d_{m}$ in the free-molecular regime using Equation (13).

$$
d_{m}^{2}=\chi_{v} d_{v e}^{2} .
$$

This provides one additional equation and reduces the underdetermination of the system of equations described in section 
"Density and Shape Factor" from 2 to 1 . Further, we can write $d_{v a}$ as a function of $d_{m}$ for this case of agglomerate particles using Equations (31) and (49):

$$
d_{v a}=\frac{\rho_{p}}{\rho_{0}} \frac{d_{m}}{\chi_{v}^{3 / 2}} .
$$

Combining Equations (5), (49), (31), and the $\rho_{\text {eff }}^{I I I}$ definition in Equation (44), it can be shown that the mass of a fractal aggregate under these conditions can be estimated as

$$
m_{p}=\frac{\pi}{6} \rho_{0} d_{m}^{2} d_{v a}=\frac{\pi}{6} \rho_{e f f}^{I I I} d_{m}^{3} .
$$

For aggregate particles with a fractal dimension of 2.0 or larger, and mobility diameters up to $600 \mathrm{~nm}$ (for $P=1 \mathrm{~atm}$ and $\lambda=65 \mathrm{~nm}$ ), Equation (51) can be used to estimate particle mass. Note that the assumption $\chi_{t} \approx \chi_{v}$ was not needed to derive Equation (51) for fractal aggregates. Equation (51) is expected to be a better estimate of particle mass for fractal aggregates than is indicated by Figure 7 for general irregular particles. The apparent discrepancy between Equation (51) and Figure 7 is due to the additional constraint for fractal aggregates discussed above. A study in which particle mass is directly measured in conjunction with both $d_{m}$ and $d_{v a}$ will allow a more complete analysis of the relative accuracy of the two methods.

\section{Dynamic Shape Factor}

An important topic for the purposes of this article is the way in which the dynamic shape factor varies for fractal aggregate particles as a function of aggregate size. Wang and Sorensen (1999) determined the ratio of the mobility radius to the radius of gyration for a large range of $\mathrm{Kn}$. Their results apply to particles with characteristics of diffusion-limited cluster aggregates (typically this means aggregates with a fractal dimension of $D_{f} \sim 1.75$ ). Baron et al. (2001b) interpreted these results in terms of the dynamic shape factor. They show two distinct regimes in which $\chi$ can be estimated from the number of primary particles, $N_{p p}$, in the aggregate:

$$
\begin{aligned}
\chi & =N_{p p}^{0.11} \\
N_{p p} & \leq 60, \\
\chi & =0.6 N_{p p}^{0.24} \\
N_{p p} & \geq 60
\end{aligned}
$$

Chan and Dahneke (1981) performed numerical calculations of the drag on straight chains of uniform spheres in the freemolecular regime. This presents a limiting case as it corresponds to a fractal aggregate with $D_{f}=1$. Their results can also be written as a functional relationship between $\chi_{v}$ and $N_{p p}$, as given by Baron et al. (2001b).

$$
\chi_{v}=\frac{\sqrt{0.802\left(N_{p p}-1\right)+1}}{N_{p p}^{1 / 3}} .
$$

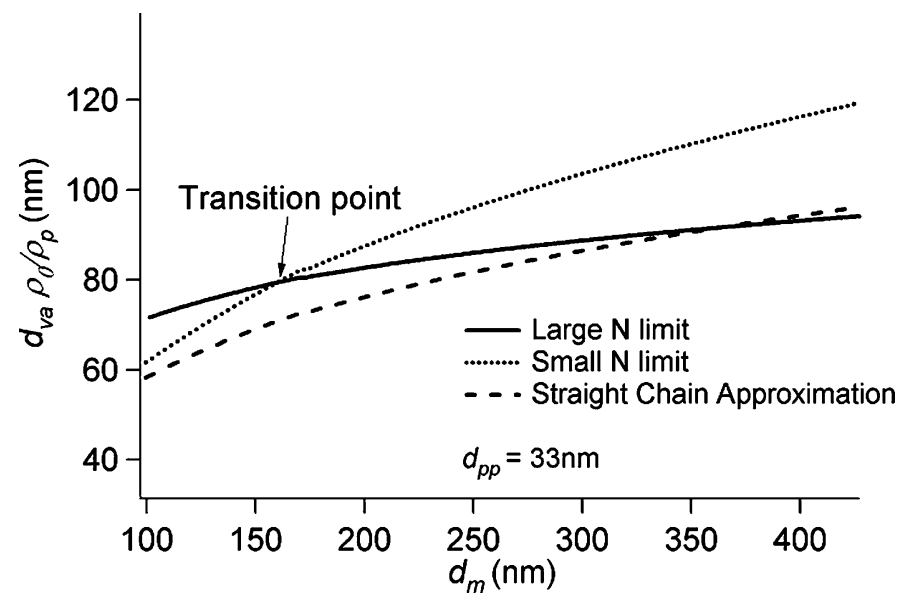

Figure 8. Relationship between $d_{v a} \rho_{0} / \rho_{p}$ and $d_{m}$ based on theoretical calculations of $\chi$ as a function of $N_{p p}$ for $N_{p p} \leq 60$ (see Equation (52)), $N_{p p} \geq 60$ (see Equation (53)) and straight chains of spherules in the free-molecular regime (see Equation (54)). As $N_{p p}$ increases, there is thought to be a smooth transition from the $N_{p p} \leq 60$ curve to the $N_{p p} \geq 60$ curve (marked by an arrow in the graph).

Given $N_{p p}$ and the primary particle diameter $\left(d_{p p}\right), d_{v e}$ can be calculated by the following relation:

$$
d_{v e}=d_{p p} N_{p p}^{1 / 3} .
$$

If $d_{p p}$ is measured (typically by electron microscopy), $d_{v e}$ can also be estimated as a function of $N_{p p}$ from Equation (55). Equation (25) and (31) can then be used to estimate $d_{m}$ and $d_{v a} \rho_{0} / \rho_{p}$. Figure 8 shows calculated, $d_{m}$ and $d_{v a} \rho_{0} / \rho_{p}$ based on the above empirical relations of $N_{p p}$ and $\chi$. For this calculation, a $d_{p p}$ of $33 \mathrm{~nm}$ was used following the measured mean diameter for diesel soot by Park et al. (2004a). In Figure 8, the dynamic shape factor of particles in the transition and continuum regimes is thought to transition smoothly from the small $N_{p p}$ limit curve to the large $N_{p p}$ limit curve as the number of primary particles grows larger than 60 (Baron et al. 2001b). The transition point is marked by an arrow on the plot.

We can also estimate and plot $\chi, d_{m}$, and $d_{v a} \rho_{0} / \rho_{p}$ as a function of $N_{p p}$, if we assume $\chi_{t} \approx \chi_{v}$ based on Equation (52), for particles with $N_{p p} \leq 60$, and based on Equation (53) for particles with $N_{p p} \geq 60$. This is a useful comparison to experimental data for fractal aggregates. Figure 9 is a plot of predicted values for $d_{m}, d_{v a} \rho_{0} / \rho_{p}$, and $\chi$ based on the above analysis of fractal aggregates, assuming a primary particle diameter of 33 $\mathrm{nm}$. Note that $d_{m}$ increases rapidly as the number of primary particles increases due to the large increase in $\chi$. Additionally, $d_{v a} \rho_{0} / \rho_{p}$ (and also $d_{v a}$, if $\rho_{p}$ is approximately constant) on the other hand is nearly constant for $N_{p p}>75$. This plot is qualitatively consistent with the experimental results of Slowik et al. (2004) shown in the companion article, and with the results of Van Gulijk et al. (2004). 


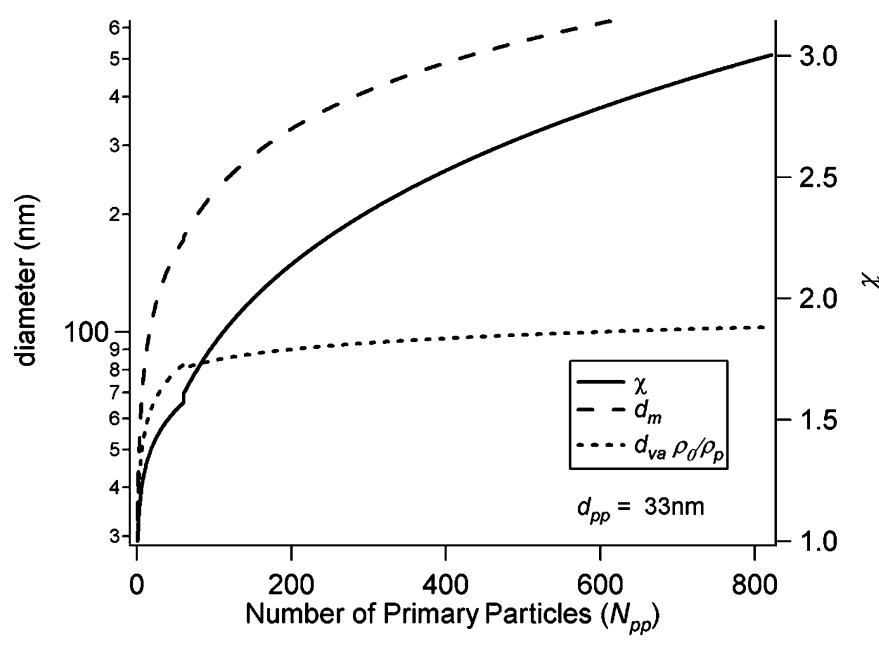

Figure 9. Theoretical calculations of $d_{v a} \rho_{0} / \rho_{p}, d_{m}$ and $\chi$, with the latter being estimated from Equations (52) and (53) for primary particles having a diameter of $33 \mathrm{~nm}$. This assumes $\chi \approx \chi_{t} \approx \chi_{v}$.

\section{Dependence of the Fractal Dimension on $\mathbf{N}_{p p}$}

The fractal dimension, as derived from the mass-mobility relationship, can be estimated as a function of $N_{p p}$ using the relationships between $\chi$ and $N_{p p}$ in Equations (52) and (53), and assuming a $d_{p p}$ of $33 \mathrm{~nm}$ as reported by Park et al. (2004a). This is shown in Figure 10 as a plot of particle volume $\left(V_{p}\right)$ versus $d_{m}$ on logarithmic axes. Based on the relationship in Equation (48), $D_{f}$ is the slope of the curve. Two distinct values of $D_{f}$ are estimated from this plot depending on the two $N_{p p}$ regimes used to estimate $\chi$ (Equations (53) and (54)). For $N_{p p} \leq 60, D_{f}=2.46$, while for $N_{p p} \geq 60, D_{f}=1.79$. The decrease in $D_{f}$ occurring

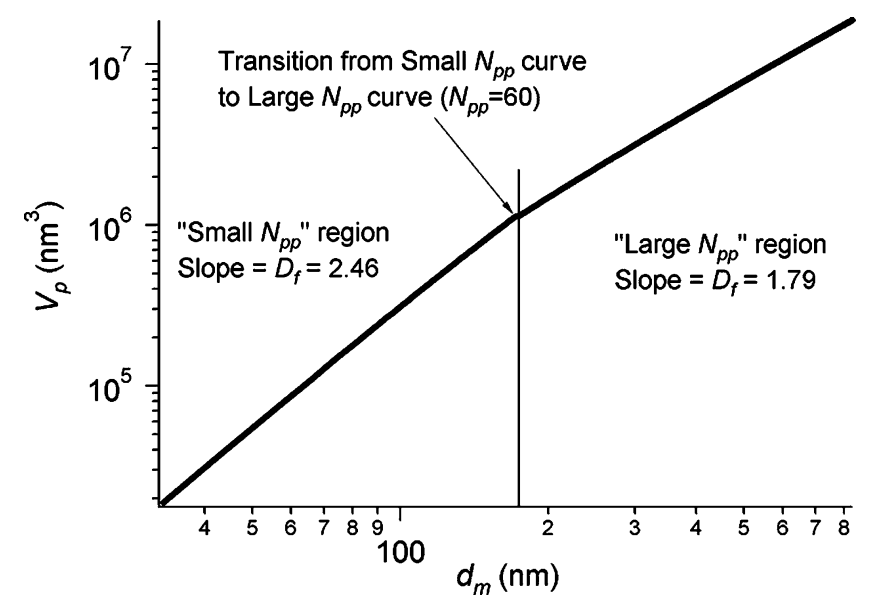

Figure 10. Particle volume versus mobility diameter for fractal agglomerates, using $\chi$ values estimated from Equations (52) and (53), assuming $\chi \approx \chi_{t} \approx \chi_{v}$ and $d_{p p}$ of $33 \mathrm{~nm}$. The slope of this log-log plot is the fractal dimension, assuming that $\rho_{p}$ is constant and that the mass-mobility relationship holds over the whole range of conditions. with the transition to the large $N_{p p}$ regime can be interpreted as being due to fractal aggregates with fewer primary particles filling a larger fraction of the volume around their center of mass than aggregates with much larger $N_{p p}$. However, note that this analysis is combining the mass-mobility relationship with values of $\chi$ determined via light scattering, and both approaches may not be fully consistent in their determination of the fractal dimension (Wang and Sorensen 1999; Van Gulijk et al. 2004). Thus this result has some uncertainty, and further research in this area is needed.

\section{Alternative Method of Estimating $D_{f}$}

An alternative method for estimating $D_{f}$ (for values of 2 or larger) can be derived using the results of Rogak et al. (1993) and Schmidt-Ott (1988). For $D_{f} \geq 2.0$, we can use Equations (47) and (51) to write

$$
d_{v a}=\frac{C^{\prime \prime \prime}}{d_{p p}^{D f}} d_{m}^{D_{f}-2} .
$$

If we further assume that the primary particle diameter is a constant value, then Equation (56) simplifies to

$$
d_{v a}=D^{\prime} d_{m}^{D_{f}-2} .
$$

Here $D^{\prime}$ is a constant. This implies that for a $D_{f}$ of $2, d_{v a}$ is a constant. Values of $D_{f}$ larger than 2 can be determined by plotting $d_{v a}$ versus $d_{m}$ on logarithmic axes ( $D_{f}$ is the slope of the resulting line). A recent paper by Van Gulijk et al. (2004) reports a similar result. For $D_{f}$ less than 2, these authors state that fractal dimension cannot be determined based on a $d_{v a}$ and $d_{m}$ measurement. They state that for fractal dimensions less than 2 , the interior spherules are no longer shielded by spherules on the exterior; consequently, drag and mass are simply functions of the number of primary particles and scale together. If that is the case, $d_{v a}$ and $d_{m}$ measurements would not contain the information needed to determine $D_{f}$ when this parameter is less than 2. This point is addressed further in the interpretation of the results from the companion article (Slowik et al. 2004).

\section{CONCLUSIONS}

Particle size measurements are expressed in terms of "equivalent diameters" that are influenced by physical morphology (shape) and density. In this work, these effective diameters are related within an analytical framework, allowing constraints to be placed on the relationships between the equivalent diameters, density, and shape (in the form of the dynamic shape factor). This framework allows important particle properties such as mass and volume to be estimated from a combination of diameter measurements.

\section{Mobility and Aerodynamic Diameters}

For irregular particles, the mobility diameter $\left(d_{m}\right)$ is always larger than the volume equivalent diameter $\left(d_{v e}\right)$. The aerodynamic diameter $\left(d_{a}\right)$ depends on particle density $\left(\rho_{p}\right)$, while $d_{m}$ 
does not. For irregular particles of standard density, $d_{a}$ is always smaller than $d_{v e}$. For a given particle, the value of the aerodynamic diameter is not unique (even for spheres), but asymptotically approaches the values of $d_{c a}$ and $d_{v a}$ in the continuum and free-molecular regimes, respectively. The dynamic shape factor also depends on flow regime. Also, $d_{m}$ depends on the pressure at which the measurement is performed for nonspherical particles.

\section{Information from Measurement of $d_{m}$ and $d_{v a}$}

Measurement of both $d_{m}$ and $d_{v a}$ allows constraints to be placed on the relationships between the dynamic shape factor, particle density, and particle mass. The particle density can be derived from $d_{m}$ and $d_{v a}$ for spherical particles. For nonspherical particles, these properties remain undetermined in the absence of additional measurements. When two additional independent measurements are available (or assumptions are made), the equations can be solved to obtain $d_{v e}, \rho_{p}$, and the dynamic shape factor in the transition and free-molecular regimes $\left(\chi_{t}\right.$ and $\chi_{v}$, respectively).

\section{Effective Density and Particle Mass}

Various definitions of effective density found in the literature have been discussed and shown to be numerically different. Consequently, intercomparison of effective densities from different studies should only be done with values calculated in the same manner. Using the relative closeness between the effective densities, $\rho_{\text {eff }}^{I}$ and $\rho_{\text {eff }}^{I I}$, and the assumption $\chi_{t} \approx \chi_{v}$, an expression is derived to estimate any submicron particle mass within about a factor of 2 based solely on measurements of $d_{v a}$ and $d_{m}$. This assumption will introduce some error, and further research on this topic is needed.

\section{Fractal Aggregates}

An extension of theoretical calculations by Wang and Sorensen (1999) for fractal aggregates is used to estimate $d_{v a}$ if density is known (or can be estimated) and the corresponding $d_{m}$ as a function of $N_{p p}$. These calculations predict two distinct values of the fractal dimension based on the mass-mobility relationship and depending on the size of the aggregate. For an aggregate with $N_{p p} \leq 60, D_{f} \sim 2.46$, while for $N_{p p} \geq 60, D_{f} \sim 1.79$. These values of the fractal dimension are consistent with those observed for some types of combustion-generated soot (Park et al. 2003; Slowik et al. 2004). Using the results from Rogak et al. (1993) allows for estimation of aggregate mass and fractal dimension for aggregates with a $D_{f} \geq 2.0$ solely from the measurement of $d_{v a}$ and $d_{m}$.

\section{REFERENCES}

Allen, M. D., Moss, O. R., and Briant, J. K. (1979). Dynamic Shape Factors for LMFBR Mixed-Oxide Fuel Aggregates, J. Aerosol Sci. 10(1):43-48.

Allen, M. D., and Raabe, O. G. (1982). Re-Evaluation of Millikan Oil Drop Data for the Motion of Small Particles in Air, J. Aerosol Sci. 13(6):537-547.
Allen, M. D., and Raabe, O. G. (1985). Slip Correction Measurements of Spherical Solid Aerosol-Particles in an Improved Millikan Apparatus, Aerosol Sci. Technol. 4(3):269-286.

Ananth, G., and Wilson, J. C. (1988). Theoretical-Analysis of the Performance of the TSI Aerodynamic Particle Sizer-The Effect of Density on Response, Aerosol Sci. Technol. 9(3):189-199.

Baron, P. A., Mazumder, M. K., and Cheng, Y. S. (2001a). Direct-Reading Techniques Using Particle Motion and Optical Detection. In Aerosol Measurement: Principles, Techniques, and Applications, edited by P. A. Baron and K. Willeke. John Wiley, New York, pp. 495-536.

Baron, P. A., Sorensen, C. M., and Brockmann, J. E. (2001b). Nonspherical Particle Measurements: Shape Factors, Fractals, and Fibers. In Aerosol Measurement: Principles, Techniques, and Applications, edited by P. A. Baron and K. Willeke. John Wiley, New York, pp. 705-749.

Baron, P. A., and Willeke, K. (2001). Gas and Particle Motion. In Aerosol Measurement: Principles, Techniques, and Applications, edited by P. A. Baron and K. Willeke. Wiley, New York, pp. 61-97.

Brockmann, J. E., and Rader, D. J. (1990). APS Response to Nonspherical Particles and Experimental-Determination of Dynamic Shape Factor, Aerosol Sci. Technol. 13(2):162-172.

Buzorius, G., Zelenyuk, A., Brechtel, F., and Imre, D. (2002). Simultaneous Determination of Individual Ambient Particle Size, Hygroscopicity and Composition, Geophys. Res. Lett. 29(20):1974.

Chakrabarti, B., Singh, M., and Sioutas, C. (2004). Development of a Near Continuous Monitor for Measurment of the Sub-150 nm PM Mass Concentration, Aerosol Sci. Technol. 38(S1):239-252.

Chan, P., and Dahneke, B. (1981). Free-Molecule Drag on Straight Chains of Uniform Spheres, J. Appl. Phy. 52(5):3106-3110.

Chen, B. T., Irwin, R., Cheng, Y. S., Hoover, M. D., and Yeh, H. C. (1993). Aerodynamic Behavior of Fiber-Like and Disk-Like Particles in a Millikan Cell Apparatus, J. Aerosol Sci. 24(2):181-195.

Cheng, Y. S. (1991). Drag Forces on Nonspherical Aerosol-Particles, Chem. Eng. Comm. 108:201-223.

Cziczo, D. J., DeMott, P. J., Brock, C., Hudson, P. K., Jesse, B., Kreidenweis, S. M., Prenni, A. J., Schreiner, J., Thomson, D. S., and Murphy, D. M. (2003). A Method for Single Particle Mass Spectrometry of Ice Nuclei, Aerosol Sci. Technol. 37(5):460-470.

Dahneke, B. (1973a). Slip Correction Factors for Nonspherical Bodies-I Introduction and Continuum Flow, J. Aerosol Sci. 4:139-145.

Dahneke, B. (1973b). Slip Correction Factors for Nonspherical Bodies-II Free Molecule Flow, J. Aerosol Sci. 4:147-161.

Dahneke, B. (1973c). Slip Correction Factors for Nonspherical Bodies-III The Form of the General Law, J. Aerosol Sci. 4:163-170.

Dick, W. D., Ziemann, P. J. Huang, P. F., and McMurry, P. H. (1998). Optical Shape Fraction Measurements of Submicrometre Laboratory and Atmospheric Aerosols, Measurement Sci. \& Technol. 9 (2):183-196.

Ehara, K., and Shin, S. (1998). Measurement of Density Distribution of Aerosol Particles by Successive Classification of Particles According to Their Mass and Diameter, J. Aerosol Sci. 29(Suppl. 1):19-20.

Emets, E. P., Kascheev, V. A., and Poluektov, P. P. (1992). A New Technique for the Determination of the Density of Airborne Particulate Matter, J. Aerosol Sci. 23(1):27-35.

Flagan, R. C. (2001). Electrical Techniques, in Aerosol Measurement: Principles, Techniques, and Applications, edited by P. A. Baron and K. Willeke, John Wiley, New York, pp. 537-568.

Friedlander, S. K. (2000). Smoke, Dust, and Haze: Fundamentals of Aerosol Dynamics. Oxford University Press, New York.

Friedlander, S. K., and Pui, D. Y. H. (2004). Emerging Issues in Nanoparticle Aerosol Sci. Technology, J. Nanoparticle Res. 6(2):313-320.

Fuchs, N. A. (1964). The Mechanics of Aerosols. Pergamon, New York.

Hand, J. L., Kreidenweis, S. M., Kreisberg, N., Hering, S., Stolzenburg, M., Dick, W., and McMurry, P. H. (2002). Comparisons of Aerosol Properties Measured by Impactors and Light Scattering from Individual Particles: Refractive Index, Number and Volume Concentrations, and Size Distributions, Atmos. Environ. 36(11):1853-1861. 
Hinds, W. C. (1999). Aerosol Technology: Properties, Behavior, and Measurement of Airborne Particles. Wiley, New York.

Huffman, J. A., Jayne, J. T., Drewnick, F., Aiken, A. C., Onasch, T., Worsnop, D. R., and Jimenez, J. L. (2004). Design, Modeling, Optimization, and Experimental Test of a Particle Beam Width Probe for the Aerodyne Aerosol Mass Spectrometer, Aerosol Sci. Tech. in preparation.

Jayne, J. T., Leard, D. C., Zhang, X. F., Davidovits, P., Smith, K. A., Kolb, C. E., and Worsnop, D. R. (2000). Development of an Aerosol Mass Spectrometer for Size and Composition Analysis of Submicron Particles, Aerosol Sci. Technol. 33(1-2):49-70.

Jimenez, J. L., Bahreini, R., Cocker, D. R., Zhuang, H., Varutbangkul, V., Flagan, R. C., Seinfeld, J. H., O'Dowd, C. D., and Hoffmann, T. (2003a). New Particle Formation from Photooxidation of Diiodomethane (CH2I2), J. Geophys. Res.-Atmos. 108(D10): 4318.

Jimenez, J. L., Bahreini, R., Cocker, D. R., Zhuang, H., Varutbangkul, V., Flagan, R. C., Seinfeld, J. H., O'Dowd, C. D., and Hoffmann, T. (2003b). New Particle Formation from Photooxidation of Diiodomethane (CH2I2), J. Geophys. Res.-Atmos. 108(D23):4733.

Jimenez, J. L., Jayne, J. T., Shi, Q., Kolb, C. E., Worsnop, D. R., Yourshaw, I., Seinfeld, J. H., Flagan, R. C., Zhang, X. F., Smith, K. A., Morris, J. W., and Davidovits, P. (2003c). Ambient Aerosol Sampling using the Aerodyne Aerosol Mass Spectrometer, J. Geophys. Res._Atmos. 108(D7):8425.

Kasper, G. (1982a). Dynamics and Measurement of Smokes. 1. Size Characterization of Non-Spherical Particles, Aerosol Sci. Technol. 1(2):187-199.

Kasper, G. (1982b). Dynamics and Measurement of Smokes. 2. The Aerodynamic Diameter of Chain Aggregates in the Transition Regime, Aerosol Sci. Technol. 1 (2):201-215.

Katrinak, K. A., Rez, P., Perkes, P. R., and Buseck, P. R. (1993). Fractal Geometry of Carbonaceous Aggregates from an Urban Aerosol, Environ. Sci. Technol. 27(3):539-547.

Kelly, W. P., and McMurry, P. H. (1992). Measurement of Particle Density by Inertial Classification of Differential Mobility Analyzer Generated Monodisperse Aerosols, Aerosol Sci. Technol. 17(3):199-212.

Khlystov, A., Stanier, C., and Pandis, S. N. (2004). An Algorithm for Combining Electrical Mobility and Aerodynamic Size Distributions Data When Measuring Ambient Aerosol, Aerosol Sci. Technol. 38(Suppl. 1):229238.

Kousaka, Y., Endo, Y., Ichitsubo, H., and Alonso, M. (1996). OrientationSpecific Dynamic Shape Factors for Doublets and Triplets of Spheres in the Transition Regime, Aerosol Sci. Technol. 24(1):36-44.

Koylu, U. O., and Faeth, G. M. (1992). Structure of Overfire Soot in Buoyant Turbulent-Diffusion Flames at Long Residence Times, Combustion Flame 89(2):140-156.

Le Bronec, E., Renoux, A., Boulaud, D., and Pourprix, M. (1999). Effect of Gravity in Differential Mobility Analysers. A New Method to Determine the Density and Mass of Aerosol Particles, J. Aerosol Sci. 30(1):89-103.

Liu, P., Ziemann, P. J., Kittelson, D. B., and McMurry, P. H. (1995a). Generating Particle Beams of Controlled Dimensions and Divergence . 1. Theory of Particle Motion in Aerodynamic Lenses and Nozzle Expansions, Aerosol Sci. Technol. 22(3):293-313.

Liu, P., Ziemann, P. J., Kittelson, D. B., and McMurry, P. H. (1995b). Generating Particle Beams of Controlled Dimensions and Divergence. 2. Experimental Evaluation of Particle Motion in Aerodynamic Lenses and Nozzle Expansions, Aerosol Sci. Technol. 22(3):314-324.

Maricq, M. M., Podsiadlik, D. H., and Chase, R. E. (2000). Size Distributions of Motor Vehicle Exhaust PM: A Comparison Between ELPI and SMPS Measurements, Aerosol Sci. Technol. 33(3):239-260.

McMurry, P. H. (2000). A Review of Atmospheric Aerosol Measurements, Atmos. Environ. 34(12-14):1959-1999.

McMurry, P. H., Wang, X., Park, K., and Ehara, K. (2002). The Relationship Between Mass and Mobility for Atmospheric Particles: A New Technique for Measuring Particle Density, Aerosol Sci. Technol. 36(2):227-238.

Morawska, L., Johnson, G., Ristovski, Z. D., and Agranovski, V. (1999). Relation Between Particle Mass and Number for Submicrometer Airborne Particles, Atmos. Environ. 33(13):1983-1990.
Park, K., Cao, F., Kittelson, D. B., and McMurry, P. H. (2003). Relationship Between Particle Mass and Mobility for Diesel Exhaust Particles, Environ. Sci. Technol. 37(3):577-583.

Park, K., Kittelson, D. B., and McMurry, P. H. (2004a). Structural Properties of Diesel Exhaust Particles Measured by Transmission Electron Microscopy (TEM): Relationships to Particle Mass and Mobility, Aerosol Sci. Technol. 38(9):881-889.

Park, K., Kittelson, D. B., Zachariah, M. R., and McMurry, P. H. (2004b). Measurement of Inherent Material Density of Nanoparticle Agglomerates, J. Nanoparticle Res. 6(2-3):267-272.

Pitz, M., Cyrys, J., Karg, E., Wiedensohler, A., Wichmann, H. E., and Heinrich, J. (2003). Variability of Apparent Particle Density of an Urban Aerosol, Environ. Sci. Technol. 37(19):4336-4342.

Rader, D. J. (1990). Momentum Slip Correction Factor for Small Particles in 9 Common Gases, J. Aerosol Sci. 21(2):161-168.

Rogak, S. N., Flagan, R. C., and Nguyen, H. V. (1993). The Mobility and Structure of Aerosol Agglomerates, Aerosol Sci. Technol. 18(1):25-47.

Schleicher, B., Kunzel, S., and Burtscher, H. (1995). In-Situ Measurement of Size and Density of Submicron Aerosol-Particles, J. Appl. Phy. 78(7):44164422.

Schmidt-Ott, A. (1988). In Situ Measurement of the Fractal Dimensionality of Ultrafine Aerosol-Particles, Appl. Phys. Lett. 52(12):954-956.

Schmidt-Ott, A., Baltensperger, U., Gaggeler, H. W., and Jost, D. T. (1990). Scaling Behavior of Physical Parameters Describing Agglomerates, J. Aerosol Sci. 21(6):711-717.

Schreiner, J., Voigt, C., Zink, P., Kohlmann, A., Knopf, D., Weisser, C., Budz, P., and Mauersberger, K. (2002). A Mass Spectrometer System for Analysis of Polar Stratospheric Aerosols, Rev. Sci. Inst. 73(2):446-452.

Slowik, J., Stainken, K., Davidovits, P., Williams, L. R., Jayne, J. T., Kolb, C. E., Worsnop, D. R., Rudich, Y., DeCarlo, P., and Jimenez, J. (2004). Particle Morphology and Density Characterization by Combined Mobility and Aerodynamic Diameter Measurements. Part 2: Application to Combustion Generated Soot Particles as a Function of Fuel Equivalence Ratio, Aerosol Sci. Technol. 38(12):1206-1222.

Stein, S. W., Turpin, B. J., Cai, X. P., Huang, C. P. F., and McMurry, P. H. (1994). Measurements of Relative Humidity-Dependent Bounce and Density for Atmospheric Particles Using the DMA-Impactor Technique, Atmos. Environ. 28(10):1739-1746.

Stober, W. (1972). Dynamic Shape Factors of Nonspherical Aerosol Particles. In Assessment of Airborne Particles; Fundamentals, Applications, and Implications to Inhalation Toxicity, edited by T. T. Mercer, P. E. Morrow, and W. Stober. Thomas, Springfield, IL: pp. xix, 540.

Tsai, C. J., Chen, S. C., Huang, C. H., and Chen, D. R. (2004). A Universal Calibration Curve for the TSI Aerodynamic Particle Sizer, Aerosol Sci. Technol. 38:467-474.

Van Gulijk, C., Marijnissen, J. C. M., Makkee, M., Moulijn, J. A., and SchmidtOtt, A. (2004). Measuring Diesel Soot with a Scanning Mobility Particle Sizer and an Electrical Low-Pressure Impactor: Performance Assessment with a Model for Fractal-like Agglomerates, J. Aerosol Sci. 35:633-655.

Virtanen, A., Ristimaki, J., and Keskinen, J. (2004). Method for Measuring Effective Density and Fractal Dimension of Aerosol Agglomerates, Aerosol Sci. Technol. 38:437-446.

Wang, G. M., and Sorensen, C. M. (1999). Diffusive Mobility of Fractal Aggregates over the Entire Knudsen Number Range, Phys. Rev. E 60(3):30363044.

Wang, H. C., and John, W. (1987). Particle Density Correction for the Aerodynamic Particle Sizer, Aerosol Sci. Technol. 6(2):191-198.

Wexler, A. S., and Johnston, M. V. (2001). Real-Time Single-Particle Analysis. In Aerosol Measurement: Principles, Techniques, and Applications, edited by P. A. Baron and K. Willeke. John Wiley, New York, pp. 365-386.

Zelenyuk, A., Cabalo, J., Baer, T., and Miller, R. E. (1999). Mass Spectrometry of Liquid Aniline Aerosol Particles by IR/UV Laser Irradiation, Anal. Chemi. 71(9):1802-1808.

Zhang, Q., Canagaratna, M. R., Jayne, J. T., Worsnop, D. R., and Jimenez, J. L. (2004a). Time and Size-Resolved Chemical Composition of Submicron 
Particles in Pittsburgh-Implications for Aerosol Sources and Processes, J. Geophys. Res.-Atmos. in press.

Zhang, X. F., Smith, K. A., Worsnop, D. R., Jimenez, J., Jayne, J. T., and Kolb, C. E. (2002). A Numerical Characterization of Particle Beam Collimation by an Aerodynamic Lens-Nozzle System: Part I. An Individual Lens or Nozzle, Aerosol Sci. Technol. 36(5):617-631.

Zhang, X. F., Smith, K. A., Worsnop, D. R., Jimenez, J., Jayne, J. T., Kolb, C. E., Morris, J., and Davidovits, P. (2004b). A Numerical Characterization of Particle Beam Collimation by an Aerodynamic Lens-Nozzle System: Part II Integrated Aerodynamic Lens-Nozzle System, Aerosol Sci. Technol. 38(6):619-638.

\section{APPENDIX A}

This appendix gives the general method for calculating $\chi_{c}$ and $\chi_{v}$ from the results of Dahneke (1973a, b). This allows the comparison of the dynamic shape factor in the continuum and free molecular regime presented in section "Relationship of $\chi$ to Flow Regime" above.

\section{Method for $\chi_{c}$ Calculation from Dahneke's (1973a) Formulation}

The drag force in the continuum regime is described in Dahneke (1973a) as

$$
F=-c_{0} \mu L_{c} V
$$

where $c_{0}$ is the resistance parameter, $\mu$ is the gas viscosity, $L_{c}$ is the characteristic length of the body, and $V$ is the relative velocity of the body with respect to the gas. The dynamic shape factor is defined in Equation (15) and can be calculated from the parameters of the Dahneke formulation as

$$
\chi_{c}=\frac{c_{0}^{\text {particle }} \cdot L_{c}}{c_{0}^{\text {sphere }} \cdot r_{v e}} .
$$

Here $L_{c}$ is the characteristic length of the body and $r_{v e}$ is the radius of a volume equivalent sphere. Dahneke (1973a) reports measured and calculated values of the orientation-averaged $c_{0}$ for discs, cylinders, spheroids, and cubes. By determining $r_{v e}$ as a function of $L_{c}$ for these simple geometric shapes, $\chi_{c}$ can be calculated.

\section{Method for $\chi_{v}$ Calculation from Dahneke's (1973b) Formulation}

Calculated values of free molecular drag are given in Dahneke (1973b) in terms of dimensionless drag, $c^{*}$ :

$$
c^{*}=-\frac{F \cdot \mathrm{Kn}}{\mu \cdot L_{c} \cdot V} .
$$

The dynamic shape factor can be calculated from the parameters of the Dahneke formulation as

$$
\chi_{v}=\frac{c_{\text {particle }}^{*} \cdot L_{c} \cdot R}{c_{\text {sphere }}^{*} \cdot r_{v e}^{2}}
$$

$R$ is the equatorial radius or semiaxis, and $r_{v e}$ is the radius of a volume equivalent sphere. For a cube both $L_{c}$ and $R$ are equal to the length of the side, $s$. Equation (A4) can be used to calculate $\chi_{v}$ from results of $c^{*}$, if the radius of a volume equivalent sphere can be expressed as function of $L_{c}$ and $R$.

Dahneke (1973b) reports calculated values of the orientationaveraged $c^{*}$ for discs, cylinders, spheroids, and cubes.

\section{Calculation of $r_{\mathrm{ve}}$ for Different Regular Particle Shapes}

Calculating $\mathrm{r}_{\mathrm{ve}}$ for a Spheroid. In the Dahneke formulation the fundamental dimensions of a spheroid for use in Equation (A4) are:

$$
\begin{aligned}
L_{c} & =\text { polar semiaxis }(a), \\
R & =\text { equatorial semiaxis }(b) .
\end{aligned}
$$

From these quantities the volume of the spheroid can be calculated in the following manner:

$$
V=\frac{4}{3} \pi a b^{2} .
$$

It follows that the radius of a volume equivalent sphere is given by

$$
r_{v e}=\left(a b^{2}\right)^{1 / 3} .
$$

Calculating $\mathrm{r}_{\mathrm{ve}}$ for a Cylinder. In the Dahneke formulation, the fundamental dimensions of a cylinder for use in Equation (A4) are

$$
\begin{aligned}
R & =\text { radius of cylinder }\left(r_{c}\right), \\
L_{c} & =\text { half height of the cylinder }(h / 2) .
\end{aligned}
$$

The volume of a cylinder is calculated in the following manner:

$$
V=\pi r_{c}^{2} h .
$$

Hence, $r_{v e}$ is given by

$$
r_{v e}=\left(\frac{3}{4} r_{c}^{2} h\right)^{1 / 3} .
$$

Calculating $\mathrm{r}_{\mathrm{ve}}$ for a Cube. The fundamental dimensions of a cube for use in Equation (A4) are

$$
\begin{aligned}
& R=L_{c}=\text { side of a cube }(s), \\
& V=s^{3} .
\end{aligned}
$$

It follows that $r_{v e}$ is given by

$$
r_{v e}=s\left(\frac{3}{4 \pi}\right)^{1 / 3} .
$$




\section{APPENDIX B}

The effect of water on particle size measurements is often a concern. The AMS and similar instruments based on lowpressure aerodynamic lenses are subject to significant losses of water in the low-pressure inlet region. (However, losses of semivolatile species such as ammonium nitrate are generally very small). For this reason it is desirable to dry the particles before they reach the lens, so that the particles do not change between the DMA and AMS measurements. Here we present an estimate of the effect of addition of water to a spherical particle on the measured value of $d_{v a}$ as a function of particle density.

Figure B1 shows estimates of "wet" $d_{v a}$ calculated using Equation (31) by continuously adding water to a spherical particle of a given density (assuming volume additivity). Figure B1 illustrates that for typical ambient aerosol particle density ( $\sim 1.5 \mathrm{~g} \mathrm{~cm}^{-3}$, e.g., Zhang et al. 2004a), or for ammonium sulfate or ammonium nitrate particles (with densities of 1.78 and $1.72 \mathrm{~g} \mathrm{~cm}^{-3}$, respectively); the addition of water to the particle has only a $\sim 10 \%$ or lower effect on the measured $d_{v a}$ for a particle where the mass of water is up to 3 times than the mass of dry material. This phenomenon is explained by the increase in particle volume being closely compensated by the decrease

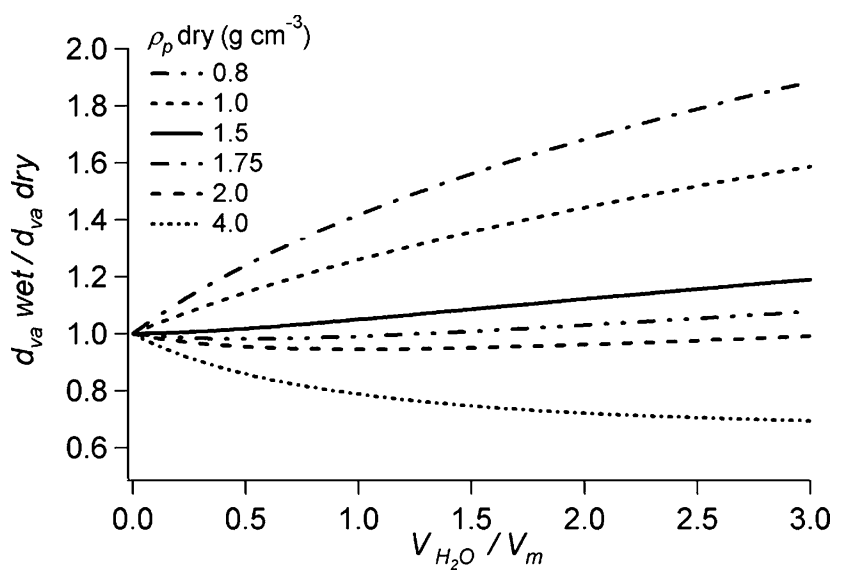

Figure B1. Ratio of wet to dry vacuum aerodynamic diameters for spherical particles, as a function of water uptake, and for several initial particle densities.

in particle density in Equation (31) in this article. In essence, this means that the measurement of $d_{v a}$ for a sulfate or nitrate dominated ambient aerosol particles is not highly sensitive to the amount of water in the particle. 


\section{Erratum}

In Aerosol Science and Technology 38(10): 1185-1205, Peter F. DeCarlo, Jay G. Slowik, Douglas R. Worsnop, Paul Davidovits, and Jose L. Jimenez, Particle Morphology and Density Characterization by Combined Mobility and Aerodynamic Diameter Measurements. Part 1: Theory, page 1196, Table 1, rows B, C, and F-G, and column "diameter relations", the following equation was published incorrectly. It should read as follows:

$$
d_{v e}=d_{m e} * \delta
$$

The publisher would like to extend an apology for any inconvenience this may have caused the authors of this article and readers of Aerosol Science and Technology. 